\title{
New Hermite-Hadamard type inequalities for $m$ and $(\alpha, m)$-convex functions on the coordinates via generalized fractional integrals
}

\author{
Seth Kermausuor \\ Alabama State University, U.S.A. \\ Received: November 2019. Accepted : June 2021
}

\begin{abstract}
In this paper, we obtained a new Hermite-Hadamard type inequality for functions of two independent variables that are $m$-convex on the coordinates via some generalized Katugampola type fractional integrals. We also established a new identity involving the second order mixed partial derivatives of functions of two independent variables via the generalized Katugampola fractional integrals. Using the identity, we established some new Hermite-Hadamard type inequalities for functions whose second order mixed partial derivatives in absolute value at some powers are $(\alpha, m)$-convex on the coordinates. Our results are extensions of some earlier results in the literature for functions of two variables.
\end{abstract}

Keywords: Hermite-Hadamard inequality, convex functions on the coordinates, $m$-convex functions on the coordinates, $(\alpha, m)$-convex functions on the coordinates, Hölder's inequality, Katugampola fractional integrals.

2010 Mathematics Subject Classification: 26A33, 26A51, 26D10, $26 D 15$. 


\section{Introduction}

The following double inequality is the well-known Hermite-Hadamard inequality:

Let $f:[a, b] \rightarrow \mathbf{R}$ be a convex function. Then the following double inequalities hold:

$$
f\left(\frac{a+b}{2}\right) \leq \frac{1}{b-a} \int_{a}^{b} f(x) d x \leq \frac{f(a)+f(b)}{2} .
$$

The Hermite-Hadamard inequality has attracted the attention of many authors in the last decades due to its numerous applications in Mathematical Analysis. Several generalizations, refinements and extensions of the Hermite-Hadamard inequality has been introduced in the literature. For some recent results related to the Hermite-Hadamard inequality, we refer the interested reader to the papers $[2,1,3,10,11,14,16,17]$. Recently, Chen and Katugampola [2] obtained the following generalizations of the Hermite-Hadamard inequality via the Katugampola fractional integrals.

Theorem 1.1. Let $\beta, \rho>0$ and $f:\left[a^{\rho}, b^{\rho}\right] \rightarrow \mathbf{R}$ be a positive function with $0 \leq a<b$. If $f$ is a convex function on $\left[a^{\rho}, b^{\rho}\right]$, then the following inequalities hold:

$$
f\left(\frac{a^{\rho}+b^{\rho}}{2}\right) \leq \frac{\rho^{\beta} \Gamma(\beta+1)}{2\left(b^{\rho}-a^{\rho}\right)^{\beta}}\left[{ }^{\rho} I_{a+}^{\beta} f\left(b^{\rho}\right)+{ }^{\rho} I_{b-}^{\beta} f\left(a^{\rho}\right)\right] \leq \frac{f\left(a^{\rho}\right)+f\left(b^{\rho}\right)}{2}
$$

where ${ }^{\rho} I_{a+}^{\beta} f$ and ${ }^{\rho} I_{b-}^{\beta} f$ are the Katugampola fractional integrals defined in Definition 1.3.

Theorem 1.2. Let $\beta, \rho>0$ and $f:\left[a^{\rho}, b^{\rho}\right] \rightarrow \mathbf{R}$ be a differentiable function on $\left(a^{\rho}, b^{\rho}\right)$ with $0 \leq a<b$. If $\left|f^{\prime}\right|$ is a convex function on $\left[a^{\rho}, b^{\rho}\right]$, then the following inequality holds:

$$
\begin{aligned}
& \left|\frac{f\left(a^{\rho}\right)+f\left(b^{\rho}\right)}{2} \quad-\frac{\rho^{\beta} \Gamma(\beta+1)}{2\left(b^{\rho}-a^{\rho}\right)^{\beta}}\left[{ }^{\rho} I_{a+}^{\beta} f\left(b^{\rho}\right)+{ }^{\rho} I_{b-}^{\beta} f\left(a^{\rho}\right)\right]\right| \\
& \leq \frac{b^{\rho}-a^{\rho}}{2\left(b^{\rho}-a^{\rho}\right)^{\beta}}\left[\left|f^{\prime}\left(a^{\rho}\right)\right|+\left|f^{\prime}\left(b^{\rho}\right)\right|\right] .
\end{aligned}
$$

Motivated by the current research on the Hermite-Hadamard inequality, our goal in this paper is to extend Theorem 1.1 for functions of two variables that are $m$-convex on the coordinates and also extend Theorem 1.2 for functions of two variables whose second order mixed partial derivatives in absolute value at certain powers are $(\alpha, m)$-convex on the coordinates. In what follows, we provide some preliminary definitions that will be useful to our work. 
Definition 1.3 (See [5]). Let $\beta, \rho>0$ and $f$ be a function a real-valued function of a single variable. The Katugampola fractional integrals of $f$ are defined as follows:

$$
{ }^{\rho} I_{a+}^{\beta} f(x)=\frac{\rho^{1-\beta}}{\Gamma(\beta)} \int_{a}^{x}\left(x^{\rho}-t^{\rho}\right)^{\beta-1} t^{\rho-1} f(t) d t
$$

and

$$
{ }^{\rho} I_{b-}^{\beta} f(x)=\frac{\rho^{1-\beta}}{\Gamma(\beta)} \int_{x}^{b}\left(t^{\rho}-x^{\rho}\right)^{\beta-1} t^{\rho-1} f(t) d t
$$

where $\Gamma(\cdot)$ is the gamma function defined by

$$
\Gamma(x)=\int_{0}^{\infty} t^{x-1} e^{-t} d t .
$$

For some recent results related to the Katugampola fractional integral, we refer the interested reader to the papers $[2,5,6,8,9,10,11]$.

The following fractional integrals for functions of two independent variables are natural extensions of the Katugampola fractional integrals in Definition 1.3.

Definition 1.4. Let $\beta_{1}, \beta_{2}, \rho_{1}, \rho_{2}>0$ and $f$ be a function of two independent variables. We define, the Katugampola fractional integrals of $f$ on the coordinates as follows:

$$
\begin{aligned}
& \rho_{1} I_{a+}^{\beta_{1}} f(x, y):=\frac{\rho_{1}-\beta_{1}}{\Gamma\left(\beta_{1}\right)} \int_{a}^{x}\left(x^{\rho_{1}}-u^{\rho_{1}}\right)^{\beta_{1}-1} u^{\rho_{1}-1} f(u, y) d u, \\
& { }^{\rho_{1}} I_{b-}^{\beta_{1}} f(x, y):=\frac{\rho_{1}^{1-\beta_{1}}}{\Gamma\left(\beta_{1}\right)} \int_{x}^{b}\left(u^{\rho_{1}}-x^{\rho_{1}}\right)^{\beta_{1}-1} u^{\rho_{1}-1} f(u, y) d u, \\
& { }_{2} I_{c+}^{\beta_{2}} f(x, y):=\frac{\rho_{2}^{1-\beta_{2}}}{\Gamma\left(\beta_{2}\right)} \int_{c}^{y}\left(y^{\rho_{2}}-v^{\rho_{2}}\right)^{\beta_{2}-1} v^{\rho_{2}-1} f(x, v) d v
\end{aligned}
$$

and

$$
{ }^{\rho_{2}} I_{d-}^{\beta_{2}} f(x, y):=\frac{\rho_{2}^{1-\beta_{2}}}{\Gamma\left(\beta_{2}\right)} \int_{y}^{d}\left(v^{\rho_{2}}-y^{\rho_{2}}\right)^{\beta_{2}-1} v^{\rho_{2}-1} f(x, v) d v .
$$

We define the Katugampola fractional integrals of $f$ in the two variables as follows:

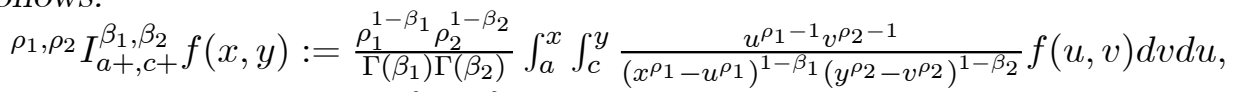

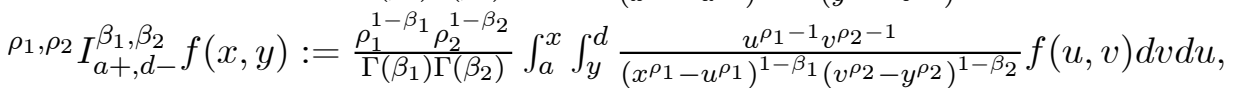

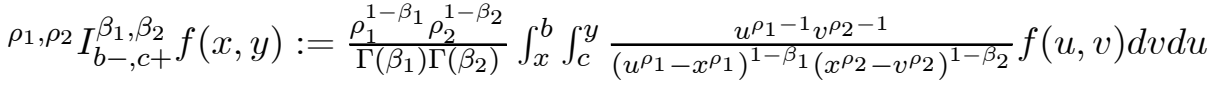

and

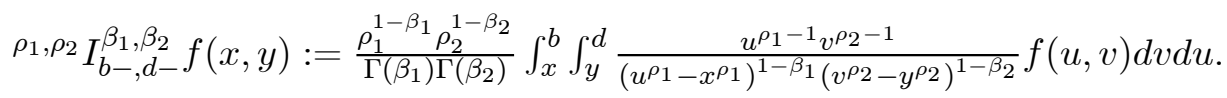


Definition 1.5 (See [1]). A function $f:[0, d] \subset \mathbf{R} \rightarrow \mathbf{R}, d>0$ is said to be $(\alpha, m)$-convex function where $(\alpha, m) \in[0,1]^{2}$ if $f(t x+m(1-t) y) \leq t^{\alpha} f(x)+m\left(1-t^{\alpha}\right) f(y)$, for all $x, y \in[0, d]$ and $t \in[0,1]$.

Remark 1.6. If we choose $(\alpha, m)=(1, m)$, then we obtain $m$-convex functions and if $(\alpha, m)=(1,1)$, then we have the ordinary convex functions.

For some recent generalizations and results related to $(\alpha, m)$-convex functions, we refer the interested reader to the papers $[1,14,17,15,7]$.

Definition 1.7 (See [16]). A function $f: \Lambda:=[0, b] \times[0, d] \rightarrow \mathbf{R}$ is said to be $m$-convex on the coordinates on $\Lambda$ for $m \in[0,1]$, if

$$
\begin{aligned}
f(t x+m(1-t) z, s y+m(1-s) w) & \leq s t f(x, y)+m t(1-s) f(x, w) \\
& +m s(1-t) f(z, y)+m^{2}(1-s)(1-t) f(z, w),
\end{aligned}
$$

for all $(x, y),(z, w) \in \Lambda$ and $(s, t) \in[0,1] \times[0,1]$. Similarly, $f: \Lambda:=$ $[0, b] \times[0, d] \rightarrow \mathbf{R}$ is said to be $(\alpha, m)$-convex on the coordinates on $\Lambda$ for $(\alpha, m) \in[0,1] \times[0,1]$, if

$$
\begin{aligned}
f(t x+m(1-t) z, s y+m(1-s) w) & \leq s^{\alpha} t^{\alpha} f(x, y)+m t^{\alpha}\left(1-s^{\alpha}\right) f(x, w) \\
& +m s^{\alpha}\left(1-t^{\alpha}\right) f(z, y)+m^{2}\left(1-s^{\alpha}\right)\left(1-t^{\alpha}\right) f(z, w),
\end{aligned}
$$

for all $(x, y),(x, w),(z, y),(z, w) \in \Lambda$ and $(s, t) \in[0,1] \times[0,1]$.

Remark 1.8. If we choose $(\alpha, m)=(1, m)$, then we obtain $m$-convex functions on the coordinates and if $(\alpha, m)=(1,1)$, then we have the concept of convex functions on the coordinates.

For some recent generalizations and results related to $(\alpha, m)$-convex functions on the coordinates, we refer the interested reader to the papers $[16,3,4,13,12]$. 


\section{Main results}

The following identities will be very useful in our main results.

Lemma 2.1. Let $\beta_{1}, \beta_{2}, \rho_{1}, \rho_{2}>0$, and $f:\left[a^{\rho_{1}}, b^{\rho_{1}}\right] \times\left[c^{\rho_{2}}, d^{\rho_{2}}\right] \rightarrow \mathbf{R}$ be a real-valued function of two independent variables. The following identities hold:

$$
\begin{aligned}
& \int_{0}^{1} s^{\beta_{2} \rho_{2}-1} f\left(x^{\rho_{1}}, s^{\rho_{2}} d^{\rho_{2}}+\left(1-s^{\rho_{2}}\right) c^{\rho_{2}}\right) d s=\frac{\rho_{2}^{\beta_{2}-1} \Gamma\left(\beta_{2}\right)}{\left(d^{\rho_{2}}-c^{\rho_{2}}\right)^{\beta_{2}}} \rho^{\rho_{2}} I_{d-}^{\beta_{2}} f\left(x^{\rho_{1}}, c^{\rho_{2}}\right), \\
& \int_{0}^{1} s^{\beta_{2} \rho_{2}-1} f\left(x^{\rho_{1}}, s^{\rho_{2}} c^{\rho_{2}}+\left(1-s^{\rho_{2}}\right) d^{\rho_{2}}\right) d s=\frac{\rho_{2}^{\beta_{2}-1} \Gamma\left(\beta_{2}\right)}{\left(d^{\rho_{2}}-c^{\rho_{2}}\right)^{\beta_{2}}} \rho_{2} I_{c+}^{\beta_{2}} f\left(x^{\rho_{1}}, d^{\rho_{2}}\right), \\
& \int_{0}^{1} t^{\beta_{1} \rho_{1}-1} f\left(t^{\rho_{1}} b^{\rho_{1}}+\left(1-t^{\rho_{1}}\right) a^{\rho_{1}}, y^{\rho_{2}}\right) d t=\frac{\rho_{1}^{\beta_{1}-1} \Gamma\left(\beta_{1}\right)}{\left(b^{\left.\rho_{1}-a^{\rho_{1}}\right)^{\beta_{1}}} \rho_{1}\right.} I_{b-}^{\beta_{1}} f\left(a^{\rho_{1}}, y^{\rho_{2}}\right) \text {, } \\
& \int_{0}^{1} t^{\beta_{1} \rho_{1}-1} f\left(t^{\rho_{1}} a^{\rho_{1}}+\left(1-t^{\rho_{1}}\right) b^{\rho_{1}}, y^{\rho_{2}}\right) d t=\frac{\rho_{1}^{\beta_{1}-1} \Gamma\left(\beta_{1}\right)}{\left(b^{\rho_{1}}-a^{\rho_{1}}\right)^{\beta_{1}}} \rho_{1} I_{a+}^{\beta_{1}} f\left(b^{\rho_{1}}, y^{\rho_{2}}\right), \\
& \int_{0}^{1} \int_{0}^{1} t^{\beta_{1} \rho_{1}-1} s^{\beta_{2} \rho_{2}-1} \quad f\left(t^{\rho_{1}} x^{\rho_{1}}+\left(1-t^{\rho_{1}}\right) b^{\rho_{1}}, s^{\rho_{2}} y^{\rho_{2}}+\left(1-s^{\rho_{2}}\right) d^{\rho_{2}}\right) d s d t \\
& =\frac{\rho_{1}^{\beta_{1}-1} \rho_{2}^{\beta_{2}-1} \Gamma\left(\beta_{1}\right) \Gamma\left(\beta_{2}\right)}{\left(b^{\rho_{1}}-x^{\rho_{1}}\right)^{\beta_{1}}\left(d^{\rho_{2}}-y^{\rho_{2}}\right)^{\beta_{2}}} \rho_{1}, \rho_{2} I_{x+, y+}^{\beta_{1}, \beta_{2}} f\left(b^{\rho_{1}}, d^{\rho_{2}}\right), \\
& \int_{0}^{1} \int_{0}^{1} t^{\beta_{1} \rho_{1}-1} s^{\beta_{2} \rho_{2}-1} \quad f\left(t^{\rho_{1}} x^{\rho_{1}}+\left(1-t^{\rho_{1}}\right) b^{\rho_{1}}, s^{\rho_{2}} y^{\rho_{2}}+\left(1-s^{\rho_{2}}\right) c^{\rho_{2}}\right) d s d t \\
& =\frac{\rho_{1}^{\beta_{1}-1} \rho_{2}^{\beta_{2}-1} \Gamma\left(\beta_{1}\right) \Gamma\left(\beta_{2}\right)}{\left(b^{\rho_{1}}-x^{\rho_{1}}\right)^{\beta_{1}}\left(y^{\rho_{2}}-c^{\rho_{2}}\right)^{\beta_{2}}}{ }^{\rho_{1}, \rho_{2}} I_{x+, y-}^{\beta_{1}, \beta_{2}} f\left(b^{\rho_{1}}, c^{\rho_{2}}\right), \\
& \int_{0}^{1} \int_{0}^{1} t^{\beta_{1} \rho_{1}-1} s^{\beta_{2} \rho_{2}-1} \quad f\left(t^{\rho_{1}} x^{\rho_{1}}+\left(1-t^{\rho_{1}}\right) a^{\rho_{1}}, s^{\rho_{2}} y^{\rho_{2}}+\left(1-s^{\rho_{2}}\right) d^{\rho_{2}}\right) d s d t \\
& =\frac{\rho_{1}^{\beta_{1}-1} \rho_{2}^{\beta_{2}-1} \Gamma\left(\beta_{1}\right) \Gamma\left(\beta_{2}\right)}{\left(x^{\rho_{1}}-a^{\rho_{1}}\right)^{\beta_{1}}\left(d^{\rho_{2}}-y^{\rho_{2}}\right)^{\beta_{2}}} \rho_{1}, \rho_{2} I_{x-, y+}^{\beta_{1}, \beta_{2}} f\left(a^{\rho_{1}}, d^{\rho_{2}}\right)
\end{aligned}
$$

and

Proof. The results follows directly by using change of variables and Definition 1.4.

Theorem 2.2. Let $\beta_{1}, \beta_{2}, \rho_{1}, \rho_{2}>0$, and $f:[0, \infty) \times[0, \infty) \rightarrow \mathbf{R}$ be an $m$-convex function on the coordinates for $m \in(0,1]$ with $0 \leq a<b$ and $0 \leq c<d$. Then the following inequalities hold: 


$$
\begin{aligned}
& \frac{1}{\beta_{1} \beta_{2} \rho_{1} \rho_{2}} f\left(\frac{a^{\rho_{1}}+b^{\rho_{1}}}{2}, \frac{c^{\rho_{2}}+d^{\rho_{2}}}{2}\right) \\
& \leq \frac{1}{4} \frac{\rho_{1}^{\beta_{1}-1} \rho_{2}^{\beta_{2}-1} \Gamma\left(\beta_{1}\right) \Gamma\left(\beta_{2}\right)}{\left(b^{\rho_{1}}-a^{\rho_{1}}\right)^{\beta_{1}}\left(d^{\rho_{2}}-c^{\rho_{2}}\right)^{\beta_{2}}} \rho^{\rho_{1}, \rho_{2}} I_{a+, c+}^{\beta_{1}, \beta_{2}} f\left(b^{\rho_{1}}, d^{\rho_{2}}\right) \\
& +\frac{m}{4} \frac{\rho_{1}^{\beta_{1}-1} \rho_{2}^{\beta_{2}-1} \Gamma\left(\beta_{1}\right) \Gamma\left(\beta_{2}\right)}{\left(b^{\rho_{1}}-a^{\rho_{1}}\right)^{\beta_{1}}\left(\frac{d^{\rho_{2}}}{m}-\frac{c^{\rho_{2}}}{m}\right)^{\beta_{2}}} \rho_{1}, \rho_{2} I_{a+,\left(\frac{d}{\rho_{2} / m}\right)-}^{\beta_{1}, \beta_{2}} f\left(b^{\rho_{1}}, \frac{c^{\rho_{2}}}{m}\right) \\
& +\frac{m}{4} \frac{\rho_{1}^{\beta_{1}-1} \rho_{2}^{\beta_{2}-1} \Gamma\left(\beta_{1}\right) \Gamma\left(\beta_{2}\right)}{\left(\frac{b^{\rho 1}}{m}-\frac{a^{\rho}}{m}\right)^{\beta_{1}}\left(d^{\rho_{2}}-c^{\rho_{2}}\right)^{\beta_{2}}} \rho_{1}, \rho_{2} I_{\left(\frac{b}{\beta_{1}}\right)-, c+}^{\beta_{1}, \beta_{2}} f\left(\frac{a^{\rho_{1}}}{m}, d^{\rho_{2}}\right) \\
& +\frac{m^{2}}{4} \frac{\rho_{1}^{\beta_{1}-1} \rho_{2}^{\beta_{2}-1} \Gamma\left(\beta_{1}\right) \Gamma\left(\beta_{2}\right)}{\left(\frac{b^{\rho}}{m}-\frac{a^{\rho}}{m}\right)^{\beta_{1}}\left(\frac{d^{\rho 2}}{m}-\frac{c^{\rho_{2}}}{m}\right)^{\beta_{2}}} \rho_{1}, \rho_{2} I_{\left(\frac{b}{\rho_{\sqrt[3]{m}}}\right)-,\left(\frac{d}{\rho_{2} / m}\right)-}^{\beta_{2}, \beta_{2}} f\left(\frac{a^{\rho_{1}}}{m}, \frac{c^{\rho_{2}}}{m}\right) \\
& \leq \frac{1}{\left(\beta_{1} \rho_{1}+\rho_{1}\right)\left(\beta_{2} \rho_{2}+\rho_{2}\right)}\left(\frac{f\left(a^{\rho_{1}}, c^{\rho_{2}}\right)}{4}+\frac{m f\left(a^{\rho_{1}}, \frac{d^{\rho_{2}}}{m}\right)}{4}+\frac{m f\left(\frac{b^{\rho_{1}}}{m}, c^{\rho_{2}}\right)}{4}\right. \\
& \left.+\frac{m^{2} f\left(\frac{b^{\rho} 1}{m}, \frac{d^{\rho} 2}{m}\right)}{4}\right) \\
& +\frac{1}{\beta_{1} \rho_{1}+\rho_{1}}\left(\frac{1}{\beta_{2} \rho_{2}}-\frac{1}{\beta_{2} \rho_{2}+\rho_{2}}\right)\left(\frac{m f\left(a^{\rho_{1}}, \frac{d^{\rho_{2}}}{m}\right)}{4}+\frac{m^{2} f\left(a^{\rho_{1}}, \frac{c^{\rho_{2}}}{m^{2}}\right)}{4}\right. \\
& \left.+\frac{m^{2} f\left(\frac{b^{\rho_{1}}}{m}, \frac{d^{\rho_{2}}}{m}\right)}{4}+\frac{m^{3} f\left(\frac{b^{\rho_{1}}}{m}, \frac{c^{\rho_{2}}}{m^{2}}\right)}{4}\right) \\
& +\frac{1}{\beta_{2} \rho_{2}+\rho_{2}}\left(\frac{1}{\beta_{1} \rho_{1}}-\frac{1}{\beta_{1} \rho_{1}+\rho_{1}}\right)\left(\frac{m f\left(\frac{b^{\rho_{1}}}{m}, c^{\rho_{2}}\right)}{4}+\frac{m^{2} f\left(\frac{b^{\rho_{1}}}{m}, \frac{d^{\rho_{2}}}{m}\right)}{4}\right. \\
& \left.+\frac{m^{2} f\left(\frac{a^{\rho_{1}}}{m^{2}}, c^{\rho_{2}}\right)}{4}+\frac{m^{3} f\left(\frac{a^{\rho_{1}}}{m^{2}}, \frac{d^{\rho_{2}}}{m}\right)}{4}\right) \\
& +\left(\frac{1}{\beta_{2} \rho_{2}}-\frac{1}{\beta_{2} \rho_{2}+\rho_{2}}\right)\left(\frac{1}{\beta_{1} \rho_{1}}-\frac{1}{\beta_{1} \rho_{1}+\rho_{1}}\right)\left(\frac{m^{2} f\left(\frac{b^{\rho_{1}}}{m}, \frac{d^{\rho_{2}}}{m}\right)}{4}+\frac{m^{3} f\left(\frac{b^{\rho_{1}}}{m}, \frac{c^{\rho_{2}}}{m^{2}}\right)}{4}\right. \\
& \left.+\frac{m^{3} f\left(\frac{a^{\rho}{ }^{2}}{m^{2}}, \frac{d^{\rho} 2}{m}\right)}{4}+\frac{m^{4} f\left(\frac{a^{\rho}}{m^{2}}, \frac{c^{\rho} 2}{m^{2}}\right)}{4}\right) .
\end{aligned}
$$

Proof. By using the $m$-convexity of $f$ on the coordinates, we have that for any $x^{\rho_{1}}, y^{\rho_{1}} \in\left[a^{\rho_{1}}, b^{\rho_{1}}\right]$ and $u^{\rho_{2}}, v^{\rho_{2}} \in\left[c^{\rho_{2}}, d^{\rho_{2}}\right]$

$$
\begin{aligned}
f\left(\frac{x^{\rho_{1}}+y^{\rho_{2}}}{2}, \frac{u_{2}^{\rho}+v^{\rho_{2}}}{2}\right) \leq & \leq \frac{1}{4} f\left(x^{\rho_{1}}, u^{\rho_{2}}\right)+\frac{m}{4} f\left(x^{\rho_{1}}, \frac{v^{\rho_{2}}}{m}\right) \\
& +\frac{m}{4} f\left(\frac{y^{\rho_{1}}}{m}, u^{\rho_{2}}\right)+\frac{m^{2}}{4} f\left(\frac{y^{\rho_{1}}}{m}, \frac{v^{\rho_{2}}}{m}\right) .
\end{aligned}
$$


In particular, if we choose $x^{\rho_{1}}=t^{\rho_{1}} a^{\rho_{1}}+\left(1-t^{\rho_{1}}\right) b^{\rho_{1}}, y^{\rho_{1}}=t^{\rho_{1}} b^{\rho_{1}}+(1-$ $\left.t^{\rho_{1}}\right) a^{\rho_{1}}, u^{\rho_{1}}=s^{\rho_{2}} c^{\rho_{2}}+\left(1-s^{\rho_{2}}\right) d^{\rho_{2}}$ and $v^{\rho_{2}}=s^{\rho_{2}} d^{\rho_{2}}+\left(1-s^{\rho_{2}}\right) c^{\rho_{2}}$ in $(2.1)$, then we have

$$
\begin{aligned}
f\left(\frac{a^{\rho_{1}}+b^{\rho_{2}}}{2}, \frac{c^{\rho_{2}}+d^{\rho_{2}}}{2}\right) & \leq \frac{1}{4} f\left(t^{\rho_{1}} a^{\rho_{1}}+\left(1-t^{\rho_{1}}\right) b^{\rho_{1}}, s^{\rho_{2}} c^{\rho_{2}}+\left(1-s^{\rho_{2}}\right) d^{\rho_{2}}\right) \\
& +\frac{m}{4} f\left(t^{\rho_{1}} a^{\rho_{1}}+\left(1-t^{\rho_{1}}\right) b^{\rho_{1}}, s^{\rho_{2}} \frac{d^{\rho_{2}}}{m}+\left(1-s^{\rho_{2}}\right) \frac{c^{\rho_{2}}}{m}\right) \\
& +\frac{m}{4} f\left(t^{\rho_{1}} \frac{b^{\rho_{1}}}{m}+\left(1-t^{\rho_{1}}\right) \frac{a^{\rho_{1}}}{m}, s^{\rho_{2}} c^{\rho_{2}}+\left(1-s^{\rho_{2}}\right) d^{\rho_{2}}\right) \\
& +\frac{m^{2}}{4} f\left(t^{\rho_{1}} \frac{b^{\rho_{1}}}{m}+\left(1-t^{\rho_{1}}\right) \frac{a^{\rho_{1}}}{m}, s^{\rho_{2}} \frac{d^{\rho_{2}}}{m}+\left(1-s^{\rho_{2}}\right) \frac{c^{\rho_{2}}}{m}\right) .
\end{aligned}
$$

Multiplying both sides of (2.2) by $t^{\beta_{1} \rho_{1}-1} s^{\beta_{2} \rho_{2}-1}$ and integrating with respect to $(s, t)$ over $[0,1] \times[0,1]$, we have

$$
\begin{aligned}
& \frac{1}{\beta_{1} \beta_{2} \rho_{1} \rho_{2}} f\left(\frac{a^{\rho_{1}}+b^{\rho_{1}}}{2}, \frac{c^{\rho_{2}}+d^{\rho_{2}}}{2}\right) \\
& \leq \frac{1}{4} \int_{0}^{1} \int_{0}^{1} t^{\beta_{1} \rho_{1}-1} s^{\beta_{2} \rho_{2}-1} f\left(t^{\rho_{1}} a^{\rho_{1}}+\left(1-t^{\rho_{1}}\right) b^{\rho_{1}}, s^{\rho_{2}} c^{\rho_{2}}+\left(1-s^{\rho_{2}}\right) d^{\rho_{2}}\right) d s d t \\
& +\frac{m}{4} \int_{0}^{1} \int_{0}^{1} t^{\beta_{1} \rho_{1}-1} s^{\beta_{2} \rho_{2}-1} f\left(t^{\rho_{1}} a^{\rho_{1}}+\left(1-t^{\rho_{1}}\right) b^{\rho_{1}}, s^{\rho_{2}} \frac{d^{\rho_{2}}}{m}+\left(1-s^{\rho_{2}}\right) \frac{c^{\rho_{2}}}{m}\right) d s d t \\
& +\frac{m}{4} \int_{0}^{1} \int_{0}^{1} t^{\beta_{1} \rho_{1}-1} s^{\beta_{2} \rho_{2}-1} f\left(t^{\rho_{1}} \frac{b^{\rho_{1}}}{m}+\left(1-t^{\rho_{1}}\right) \frac{a^{\rho_{1}}}{m}, s^{\rho_{2}} c^{\rho_{2}}+\left(1-s^{\rho_{2}}\right) d^{\rho_{2}}\right) d s d t \\
& +\frac{m^{2}}{4} \int_{0}^{1} \int_{0}^{1} t^{\beta_{1} \rho_{1}-1} s^{\beta_{2} \rho_{2}-1} f\left(t^{\rho_{1}} \frac{b^{\rho_{1}}}{m}+\left(1-t^{\rho_{1}}\right) \frac{a^{\rho_{1}}}{m}, s^{\rho_{2}} \frac{d^{\rho_{2}}}{m}+\left(1-s^{\rho_{2}}\right) \frac{c^{\rho_{2}}}{m}\right) d s d t .
\end{aligned}
$$

Now, by using Lemma 2.1 with some slight modifications and (2.3), we have

$$
\begin{aligned}
& \frac{1}{\beta_{1} \beta_{2} \rho_{1} \rho_{2}} f\left(\frac{a^{\rho_{1}}+b^{\rho_{2}}}{2}, \frac{c^{\rho_{2}}+d^{\rho_{2}}}{2}\right) \\
& \leq \frac{1}{4} \frac{\rho_{1}^{\beta_{1}-1} \rho_{2}^{\beta_{2}-1} \Gamma\left(\beta_{1}\right) \Gamma\left(\beta_{2}\right)}{\left(b^{\rho_{1}}-a^{\rho_{1}}\right)^{\beta_{1}}\left(d^{\rho_{2}}-c^{\rho_{2}}\right)^{\beta_{2}}} \rho_{1}, \rho_{2} I_{a+, c+}^{\beta_{1}, \beta_{2}} f\left(b^{\rho_{1}}, d^{\rho_{2}}\right) \\
& +\frac{m}{4} \frac{\rho_{1}^{\beta_{1}-1} \rho_{2}^{\beta_{2}-1} \Gamma\left(\beta_{1}\right) \Gamma\left(\beta_{2}\right)}{\left(b^{\rho_{1}}-a^{\rho_{1}}\right)^{\beta_{1}}\left(\frac{d^{\rho} 2}{m}-\frac{c^{\rho_{2}}}{m}\right)^{\beta_{2}}} \rho_{1}, \rho_{2} I_{a+,\left(\frac{d}{\rho 2}{ }^{\beta_{m}}\right)-}^{\beta_{1}, \beta_{2}} f\left(b^{\rho_{1}}, \frac{c^{\rho_{2}}}{m}\right) \\
& +\frac{m}{4} \frac{\rho_{1}^{\beta_{1}-1} \rho_{2}^{\beta_{2}-1} \Gamma\left(\beta_{1}\right) \Gamma\left(\beta_{2}\right)}{\left(\frac{b^{1} 1}{m}-\frac{a^{\rho 1}}{m}\right)^{\beta_{1}}\left(d^{\rho_{2}}-c^{\rho_{2}}\right)^{\beta_{2}}} \rho_{1}, \rho_{2} I_{\left(\frac{b}{\rho_{\sqrt[m]{m}}}\right)-, c+}^{\beta_{1}, \beta_{2}} f\left(\frac{a^{\rho_{1}}}{m}, d^{\rho_{2}}\right) \\
& +\frac{m^{2}}{4} \frac{\rho_{1}^{\beta_{1}-1} \rho_{2}^{\beta_{2}-1} \Gamma\left(\beta_{1}\right) \Gamma\left(\beta_{2}\right)}{\left(\frac{b^{\rho} 1}{m}-\frac{a^{\rho} 1}{m}\right)^{\beta_{1}}\left(\frac{d^{\rho} 2}{m}-\frac{c^{\rho_{2}}}{m}\right)^{\beta_{2}}} \rho_{1}, \rho_{2} I_{\left(\frac{b}{\rho_{1}}\right)-,\left(\frac{d}{\rho_{2} / m}\right)-}^{\beta_{1}, \beta_{2}} f\left(\frac{a^{\rho_{1}}}{m}, \frac{c^{\rho_{2}}}{m}\right)
\end{aligned}
$$

This proves the first inequality. On the other hand, by using the fact that $f$ is $m$-convex on the coordinates, we have 


$$
\begin{aligned}
& \int_{0}^{1} \int_{0}^{1} t^{\beta_{1} \rho_{1}-1} s^{\beta_{2} \rho_{2}-1} f\left(t^{\rho_{1}} a^{\rho_{1}}+\left(1-t^{\rho_{1}}\right) b^{\rho_{1}}, s^{\rho_{2}} c^{\rho_{2}}+\left(1-s^{\rho_{2}}\right) d^{\rho_{2}}\right) d s d t \\
& \leq f\left(a^{\rho_{1}}, c^{\rho_{2}}\right) \int_{0}^{1} \int_{0}^{1} t^{\beta_{1} \rho_{1}-1} s^{\beta_{2} \rho_{2}-1} s^{\rho_{2}} t^{\rho_{1}} d s d t \\
& +m f\left(a^{\rho_{1}}, \frac{d^{\rho_{2}}}{m}\right) \int_{0}^{1} \int_{0}^{1} t^{\beta_{1} \rho_{1}-1} s^{\beta_{2} \rho_{2}-1}\left(1-s^{\rho_{2}}\right) t^{\rho_{1}} d s d t \\
& +m f\left(\frac{b^{\rho_{1}}}{m}, c^{\rho_{2}}\right) \int_{0}^{1} \int_{0}^{1} t^{\beta_{1} \rho_{1}-1} s^{\beta_{2} \rho_{2}-1} s^{\rho_{2}}\left(1-t^{\rho_{1}}\right) d s d t \\
& +m^{2} f\left(\frac{b^{\rho_{1}}}{m}, \frac{d^{\rho_{2}}}{m}\right) \int_{0}^{1} \int_{0}^{1} t^{\beta_{1} \rho_{1}-1} s^{\beta_{2} \rho_{2}-1}\left(1-s^{\rho_{2}}\right)\left(1-t^{\rho_{1}}\right) d s d t .
\end{aligned}
$$

That is,

$$
\begin{aligned}
& \int_{0}^{1} \int_{0}^{1} t^{\beta_{1} \rho_{1}-1} s^{\beta_{2} \rho_{2}-1} f\left(t^{\rho_{1}} a^{\rho_{1}}+\left(1-t^{\rho_{1}}\right) b^{\rho_{1}}, s^{\rho_{2}} c^{\rho_{2}}+\left(1-s^{\rho_{2}}\right) d^{\rho_{2}}\right) d s d t \\
& \leq f\left(a^{\rho_{1}}, c^{\rho_{2}}\right) \frac{1}{\left(\beta_{1} \rho_{1}+\rho_{1}\right)\left(\beta_{2} \rho_{2}+\rho_{2}\right)} \\
& +m f\left(a^{\rho_{1}}, \frac{d^{\rho_{2}}}{m}\right) \frac{1}{\beta_{1} \rho_{1}+\rho_{1}}\left(\frac{1}{\beta_{2} \rho_{2}}-\frac{1}{\beta_{2} \rho_{2}+\rho_{2}}\right) \\
& +m f\left(\frac{b^{\rho_{1}}}{m}, c^{\rho_{2}}\right) \frac{1}{\beta_{2} \rho_{2}+\rho_{2}}\left(\frac{1}{\beta_{1} \rho_{1}}-\frac{1}{\beta_{1} \rho_{1}+\rho_{1}}\right) \\
& +m^{2} f\left(\frac{b^{\rho_{1}}}{m}, \frac{d^{\rho_{2}}}{m}\right)\left(\frac{1}{\beta_{2} \rho_{2}}-\frac{1}{\beta_{2} \rho_{2}+\rho_{2}}\right)\left(\frac{1}{\beta_{1} \rho_{1}}-\frac{1}{\beta_{1} \rho_{1}+\rho_{1}}\right) .
\end{aligned}
$$

Similarly, we have

$$
\begin{aligned}
& \int_{0}^{1} \int_{0}^{1} t^{\beta_{1} \rho_{1}-1} s^{\beta_{2} \rho_{2}-1} f\left(t^{\rho_{1}} a^{\rho_{1}}+\left(1-t^{\rho_{1}}\right) b^{\rho_{1}}, s^{\rho_{2}} \frac{d^{\rho_{2}}}{m}+\left(1-s^{\rho_{2}}\right) \frac{c^{\rho_{2}}}{m}\right) d s d t \\
& \leq f\left(a^{\rho_{1}}, \frac{d^{\rho_{2}}}{m}\right) \frac{1}{\left(\beta_{1} \rho_{1}+\rho_{1}\right)\left(\beta_{2} \rho_{2}+\rho_{2}\right)} \\
& +m f\left(a^{\rho_{1}}, \frac{c^{\rho_{2}}}{m^{2}}\right) \frac{1}{\beta_{1} \rho_{1}+\rho_{1}}\left(\frac{1}{\beta_{2} \rho_{2}}-\frac{1}{\beta_{2} \rho_{2}+\rho_{2}}\right) \\
& +m f\left(\frac{b^{\rho_{1}}}{m}, \frac{d^{\rho_{2}}}{m}\right) \frac{1}{\beta_{2} \rho_{2}+\rho_{2}}\left(\frac{1}{\beta_{1} \rho_{1}}-\frac{1}{\beta_{1} \rho_{1}+\rho_{1}}\right) \\
& +m^{2} f\left(\frac{b^{\rho_{1}}}{m}, \frac{c^{\rho_{2}}}{m^{2}}\right)\left(\frac{1}{\beta_{2} \rho_{2}}-\frac{1}{\beta_{2} \rho_{2}+\rho_{2}}\right)\left(\frac{1}{\beta_{1} \rho_{1}}-\frac{1}{\beta_{1} \rho_{1}+\rho_{1}}\right),
\end{aligned}
$$

$$
\begin{aligned}
& \int_{0}^{1} \int_{0}^{1} t^{\beta_{1} \rho_{1}-1} s^{\beta_{2} \rho_{2}-1} f\left(t^{\rho_{1}} \frac{b^{\rho_{1}}}{m}+\left(1-t^{\rho_{1}}\right) \frac{a_{1}^{\rho}}{m}, s^{\rho_{2}} c^{\rho_{2}}+\left(1-s^{\rho_{2}}\right) d^{\rho_{2}}\right) d s d t \\
& \leq f\left(\frac{b^{\rho_{1}}}{m}, c^{\rho_{2}}\right) \frac{1}{\left(\beta_{1} \rho_{1}+\rho_{1}\right)\left(\beta_{2} \rho_{2}+\rho_{2}\right)} \\
& +m f\left(\frac{b^{\rho_{1}}}{m}, \frac{d^{\rho_{2}}}{m}\right) \frac{1}{\beta_{1} \rho_{1}+\rho_{1}}\left(\frac{1}{\beta_{2} \rho_{2}}-\frac{1}{\beta_{2} \rho_{2}+\rho_{2}}\right) \\
& +m f\left(\frac{a^{\rho_{1}}}{m^{2}}, c^{\rho_{2}}\right) \frac{1}{\beta_{2} \rho_{2}+\rho_{2}}\left(\frac{1}{\beta_{1} \rho_{1}}-\frac{1}{\beta_{1} \rho_{1}+\rho_{1}}\right) \\
& +m^{2} f\left(\frac{a^{\rho_{1}}}{m^{2}}, \frac{d^{\rho_{2}}}{m}\right)\left(\frac{1}{\beta_{2} \rho_{2}}-\frac{1}{\beta_{2} \rho_{2}+\rho_{2}}\right)\left(\frac{1}{\beta_{1} \rho_{1}}-\frac{1}{\beta_{1} \rho_{1}+\rho_{1}}\right)
\end{aligned}
$$

and 


$$
\begin{aligned}
& \int_{0}^{1} \int_{0}^{1} t^{\beta_{1} \rho_{1}-1} s^{\beta_{2} \rho_{2}-1} f\left(t^{\rho_{1}} \frac{b^{\rho_{1}}}{m}+\left(1-t^{\rho_{1}}\right) \frac{a^{\rho_{1}}}{m}, s^{\rho_{2}} \frac{d^{\rho_{2}}}{m}+\left(1-s^{\rho_{2}}\right) \frac{c^{\rho_{2}}}{m}\right) d s d t \\
& \leq f\left(\frac{b^{\rho_{1}}}{m}, \frac{d^{\rho_{2}}}{m}\right) \frac{1}{\left(\beta_{1} \rho_{1}+\rho_{1}\right)\left(\beta_{2} \rho_{2}+\rho_{2}\right)} \\
& +m f\left(\frac{b^{\rho_{1}}}{m}, \frac{c^{\rho_{2}}}{m^{2}}\right) \frac{1}{\beta_{1} \rho_{1}+\rho_{1}}\left(\frac{1}{\beta_{2} \rho_{2}}-\frac{1}{\beta_{2} \rho_{2}+\rho_{2}}\right) \\
& +m f\left(\frac{a^{\rho_{1}}}{m^{2}}, \frac{d^{\rho_{2}}}{m}\right) \frac{1}{\beta_{2} \rho_{2}+\rho_{2}}\left(\frac{1}{\beta_{1} \rho_{1}}-\frac{1}{\beta_{1} \rho_{1}+\rho_{1}}\right) \\
& +m^{2} f\left(\frac{a^{\rho_{1}}}{m^{2}}, \frac{c^{\rho_{2}}}{m^{2}}\right)\left(\frac{1}{\beta_{2} \rho_{2}}-\frac{1}{\beta_{2} \rho_{2}+\rho_{2}}\right)\left(\frac{1}{\beta_{1} \rho_{1}}-\frac{1}{\beta_{1} \rho_{1}+\rho_{1}}\right)
\end{aligned}
$$

Now, we multiply (2.4) by $\frac{1}{4}$, multiply (2.5) and (2.6) by $\frac{m}{4}$, and multiply $(2.7)$ by $\frac{m^{2}}{4}$, then add the results and use Lemma 2.1 to get

$$
\begin{aligned}
& \frac{1}{4} \frac{\rho_{1}^{\beta_{1}-1} \rho_{2}^{\beta_{2}-1} \Gamma\left(\beta_{1}\right) \Gamma\left(\beta_{2}\right)}{\left(b^{\rho_{1}}-a^{\rho_{1}}\right)^{\beta_{1}}\left(d^{\rho_{2}}-c^{\rho_{2}}\right)^{\beta_{2}}} \rho_{1}, \rho_{2} I_{a+, c+}^{\beta_{1}, \beta_{2}} f\left(b^{\rho_{1}}, d^{\rho_{2}}\right)
\end{aligned}
$$

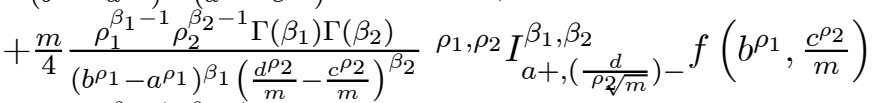

$$
\begin{aligned}
& +\frac{m}{4} \frac{\rho_{1}^{\beta_{1}-1} \rho_{2}^{\beta_{2}-1} \Gamma\left(\beta_{1}\right) \Gamma\left(\beta_{2}\right)}{\left(\frac{b^{\rho} \rho_{1}}{m}-\frac{a^{\rho}{ }_{1}}{m}\right)^{\beta_{1}}\left(d^{\rho_{2}}-c^{\rho_{2}}\right)^{\beta_{2}}} \rho_{1}, \rho_{2} I_{\left(\frac{b}{\rho_{1}^{m}}\right)-, c+}^{\beta_{1}, \beta_{2}} f\left(\frac{a^{\rho_{1}}}{m}, d^{\rho_{2}}\right) \\
& +\frac{m^{2}}{4} \frac{\rho_{1}^{\beta_{1}-1} \rho_{2}^{\beta_{2}-1} \Gamma\left(\beta_{1}\right) \Gamma\left(\beta_{2}\right)}{\left(\frac{b^{\rho} 1}{m}-\frac{a^{\rho}}{m}\right)^{\beta_{1}}\left(\frac{d^{\rho 2}}{m}-\frac{c^{\rho} 2}{m}\right)^{\beta_{2}}} \rho_{1}, \rho_{2} I_{\left(\frac{b}{\rho_{1}}\right)-,\left(\frac{d}{\rho_{2} / m}\right)-}^{\beta_{1}, \beta_{2}} f\left(\frac{a^{\rho_{1}}}{m}, \frac{c^{\rho_{2}}}{m}\right) \\
& \leq \frac{1}{\left(\beta_{1} \rho_{1}+\rho_{1}\right)\left(\beta_{2} \rho_{2}+\rho_{2}\right)}\left(\frac{f\left(a^{\rho_{1}}, c^{\rho_{2}}\right)}{4}+\frac{m f\left(a^{\rho_{1}}, \frac{d^{\rho_{2}}}{m}\right)}{4}+\frac{m f\left(\frac{b^{\rho_{1}}}{m}, c^{\rho_{2}}\right)}{4}\right. \\
& \left.+\frac{m^{2} f\left(\frac{b^{\rho_{1}}}{m}, \frac{d^{\rho_{2}}}{m}\right)}{4}\right) \\
& +\frac{1}{\beta_{1} \rho_{1}+\rho_{1}}\left(\frac{1}{\beta_{2} \rho_{2}}-\frac{1}{\beta_{2} \rho_{2}+\rho_{2}}\right)\left(\frac{m f\left(a^{\rho_{1}}, \frac{d^{\rho_{2}}}{m}\right)}{4}+\frac{m^{2} f\left(a^{\rho_{1}}, \frac{c^{\rho_{2}}}{m^{2}}\right)}{4}\right. \\
& \left.+\frac{m^{2} f\left(\frac{b^{\rho_{1}}}{m}, \frac{d^{\rho_{2}}}{m}\right)}{4}+\frac{m^{3} f\left(\frac{b^{\rho_{1}}}{m}, \frac{c^{\rho_{2}}}{m^{2}}\right)}{4}\right) \\
& +\frac{1}{\beta_{2} \rho_{2}+\rho_{2}}\left(\frac{1}{\beta_{1} \rho_{1}}-\frac{1}{\beta_{1} \rho_{1}+\rho_{1}}\right)\left(\frac{m f\left(\frac{b^{\rho_{1}}}{m}, c^{\rho_{2}}\right)}{4}+\frac{m^{2} f\left(\frac{b^{\rho_{1}}}{m}, \frac{d^{\rho_{2}}}{m}\right)}{4}\right. \\
& \left.+\frac{m^{2} f\left(\frac{a^{\rho_{1}}}{m^{2}}, c^{\rho_{2}}\right)}{4}+\frac{m^{3} f\left(\frac{a^{\rho_{1}}}{m^{2}}, \frac{d^{\rho_{2}}}{m}\right)}{4}\right) \\
& +\left(\frac{1}{\beta_{2} \rho_{2}}-\frac{1}{\beta_{2} \rho_{2}+\rho_{2}}\right)\left(\frac{1}{\beta_{1} \rho_{1}}-\frac{1}{\beta_{1} \rho_{1}+\rho_{1}}\right)\left(\frac{m^{2} f\left(\frac{b^{\rho_{1}}}{m}, \frac{d^{\rho_{2}}}{m}\right)}{4}+\frac{m^{3} f\left(\frac{b^{\rho_{1}}}{m}, \frac{\rho^{\rho_{2}}}{m^{2}}\right)}{4}\right. \\
& \left.+\frac{m^{3} f\left(\frac{a^{\rho}{ }_{1}}{m^{2}}, \frac{d^{\rho} 2}{m}\right)}{4}+\frac{m^{4} f\left(\frac{a^{\rho}{ }^{2}}{m^{2}}, \frac{c^{\rho} 2}{m^{2}}\right)}{4}\right) .
\end{aligned}
$$

This proves the second inequality. Hence the proof is complete. 
Corollary 2.3. Let $\beta_{1}, \beta_{2}, \rho_{1}, \rho_{2}>0$, and $f:\left[a^{\rho_{1}}, b^{\rho_{1}}\right] \times\left[c^{\rho_{2}}, d^{\rho_{2}}\right] \rightarrow \mathbf{R}$ be a convex function on the coordinates with $0 \leq a<b$ and $0 \leq c<d$. Then the following inequalities hold:

$$
\begin{aligned}
& f\left(\frac{a^{\rho_{1}}+b^{\rho_{2}}}{2}, \frac{c^{\rho_{2}}+d^{\rho_{2}}}{2}\right) \\
& \leq \frac{1}{4} \frac{\rho_{1}^{\beta_{1}} \rho_{2}^{\beta_{2}} \Gamma\left(\beta_{1}+1\right) \Gamma\left(\beta_{2}+1\right)}{\left(b^{\rho_{1}}-a^{\rho_{1}}\right)^{\beta_{1}}\left(d^{\rho_{2}}-c^{\rho_{2}}\right)^{\beta_{2}}}\left({ }^{\rho_{1}, \rho_{2}} I_{b-, d-}^{\beta_{1}, \beta_{2}} f\left(a^{\rho_{1}}, c^{\rho_{2}}\right)+{ }^{\rho_{1}, \rho_{2}} I_{b-, c+}^{\beta_{1}, \beta_{2}} f\left(a^{\rho_{1}}, d^{\rho_{2}}\right)\right. \\
& \left.+{ }^{\rho_{1}, \rho_{2}} I_{a+, d-}^{\beta_{1}, \beta_{2}} f\left(b^{\rho_{1}}, c^{\rho_{2}}\right)+{ }^{\rho_{1}, \rho_{2}} I_{a+, c+}^{\beta_{1}, \beta_{2}} f\left(b^{\rho_{1}}, d^{\rho_{2}}\right)\right)
\end{aligned}
$$

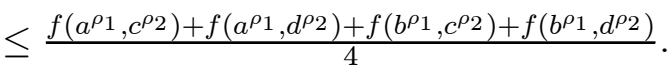

Proof. The result follows directly from Theorem 2.2 if we choose $m=1$.

Lemma 2.4. Let $\beta_{1}, \beta_{2}, \rho_{1}, \rho_{2}>0$ and $f:\left[a^{\rho_{1}}, b^{\rho_{1}}\right] \times\left[c^{\rho_{2}}, d^{\rho_{2}}\right] \rightarrow \mathbf{R}$ be a twice partial differentiable mapping on $\left(a^{\rho_{1}}, b^{\rho_{1}}\right) \times\left(c^{\rho_{2}}, d^{\rho_{2}}\right)$ with $0 \leq a<b$ and $0 \leq c<d$, and $\frac{\partial^{2} f}{\partial t \partial s} \in L_{1}\left(\left[a^{\rho_{1}}, b^{\rho_{1}}\right] \times\left[c^{\rho_{2}}, d^{\rho_{2}}\right]\right)$. Then the following equality holds:



$$
\begin{aligned}
& -\frac{\rho_{2}^{\beta_{2}} \Gamma\left(\beta_{2}+1\right)}{4\left(d^{\rho_{2}}-c^{\rho_{2}}\right)^{\beta_{2}}}\left[{ }^{\rho_{2}} I_{c+}^{\beta_{2}} f\left(a^{\rho_{1}}, d^{\rho_{2}}\right)+{ }^{\rho_{2}} I_{c+}^{\beta_{2}} f\left(b^{\rho_{1}}, d^{\rho_{2}}\right)\right. \\
& \left.+^{\rho_{2}} I_{d-}^{\beta_{2}} f\left(a^{\rho_{1}}, c^{\rho_{2}}\right)+{ }^{\rho_{2}} I_{d-}^{\beta_{2}} f\left(b^{\rho_{1}}, c^{\rho_{2}}\right)\right] \\
& -\frac{\rho_{1}^{\beta_{1}} \Gamma\left(\beta_{1}+1\right)}{4\left(b^{\rho_{1}}-a^{\rho_{1}}\right)^{\beta_{1}}}\left[\rho^{\rho_{1}} I_{a+}^{\beta_{1}} f\left(b^{\rho_{1}}, c^{\rho_{2}}\right)+{ }^{\rho_{1}} I_{a+}^{\beta_{1}} f\left(b^{\rho_{1}}, d^{\rho_{2}}\right)\right. \\
& \left.+{ }^{\rho_{1}} I_{b-}^{\beta_{1}} f\left(a^{\rho_{1}}, c^{\rho_{2}}\right)+{ }^{\rho_{1}} I_{b-}^{\beta_{1}} f\left(a^{\rho_{1}}, d^{\rho_{2}}\right)\right] \\
& +\frac{\rho_{1}^{\beta_{1}} \rho_{2}^{\beta_{2}} \Gamma\left(\beta_{1}+1\right) \Gamma\left(\beta_{2}+1\right)}{4\left(b^{\rho_{1}}-a^{\rho_{1}}\right)^{\beta_{1}}\left(d^{\rho_{2}}-c^{\rho_{2}}\right)^{\beta_{2}}}\left[{ }^{\rho_{1}, \rho_{2}} I_{a+, c+}^{\beta_{1}, \beta_{2}} f\left(b^{\rho_{1}}, d^{\rho_{2}}\right)+{ }^{\rho_{1}, \rho_{2}} I_{a+, d-}^{\beta_{1}, \beta_{2}} f\left(b^{\rho_{1}}, c^{\rho_{2}}\right)\right. \\
& \left.+^{\rho_{1}, \rho_{2}} I_{b-, c+}^{\beta_{1}, \beta_{2}} f\left(c^{\rho_{1}}, d^{\rho_{2}}\right)+{ }^{\rho_{1}, \rho_{2}} I_{b-, d-}^{\beta_{1}, \beta_{2}} f\left(a^{\rho_{1}}, c^{\rho_{2}}\right)\right] \\
& =\frac{\rho_{1} \rho_{2}\left(b^{\rho_{1}}-a^{\rho_{1}}\right)\left(d^{\left.\rho_{2}-c^{\rho_{2}}\right)}\right.}{4}\left(I_{1}-I_{2}-I_{3}+I_{4}\right),
\end{aligned}
$$

where 


$$
\begin{gathered}
I_{1}=\int_{0}^{1} \int_{0}^{1} s^{\left(\beta_{2}+1\right) \rho_{2}-1} t^{\left(\beta_{1}+1\right) \rho_{1}-1} \frac{\partial^{2}}{\partial t \partial s} f\left(t^{\rho_{1}} b^{\rho_{1}}+\left(1-t^{\rho_{1}}\right) a^{\rho_{1}}, s^{\rho_{2}} d^{\rho_{2}}+\left(1-s^{\rho_{2}}\right) c^{\rho_{2}}\right) d t d s, \\
I_{2}=\int_{0}^{1} \int_{0}^{1} s^{\left(\beta_{2}+1\right) \rho_{2}-1} t^{\left(\beta_{1}+1\right) \rho_{1}-1} \frac{\partial^{2}}{\partial t \partial s} f\left(t^{\rho_{1}} b^{\rho_{1}}+\left(1-t^{\rho_{1}}\right) a^{\rho_{1}}, s^{\rho_{2}} c^{\rho_{2}}+\left(1-s^{\rho_{2}}\right) d^{\rho_{2}}\right) d t d s, \\
I_{3}=\int_{0}^{1} \int_{0}^{1} s^{\left(\beta_{2}+1\right) \rho_{2}-1} t^{\left(\beta_{1}+1\right) \rho_{1}-1} \frac{\partial^{2}}{\partial t \partial s} f\left(t^{\rho_{1}} a^{\rho_{1}}+\left(1-t^{\rho_{1}}\right) b^{\rho_{1}}, s^{\rho_{2}} d^{\rho_{2}}+\left(1-s^{\rho_{2}}\right) c^{\rho_{2}}\right) d t d s
\end{gathered}
$$
and

$$
I_{4}=\int_{0}^{1} \int_{0}^{1} s^{\left(\beta_{2}+1\right) \rho_{2}-1} t^{\left(\beta_{1}+1\right) \rho_{1}-1} \frac{\partial^{2}}{\partial t \partial s} f\left(t^{\rho_{1}} a^{\rho_{1}}+\left(1-t^{\rho_{1}}\right) b^{\rho_{1}}, s^{\rho_{2}} c^{\rho_{2}}+\left(1-s^{\rho_{2}}\right) d^{\rho_{2}}\right) d t d s .
$$

Proof. By using integration by parts, we have

$$
\begin{aligned}
& I_{1}=\int_{0}^{1} s^{\left(\beta_{2}+1\right) \rho_{2}-1}\left[\int_{0}^{1} t^{\beta_{1} \rho_{1}} t^{\rho_{1}-1} \frac{\partial^{2}}{\partial t \partial s} f\left(t^{\rho_{1}} b^{\rho_{1}}+\left(1-t^{\rho_{1}}\right) a^{\rho_{1}}, s^{\rho_{2}} d^{\rho_{2}}+\left(1-s^{\rho_{2}}\right) c^{\rho_{2}}\right) d t\right] d s \\
& =\int_{0}^{1} s^{\left(\beta_{2}+1\right) \rho_{2}-1}\left[\left.\frac{1}{\left(b^{\rho_{1}}-a^{\rho_{1}}\right) \rho_{1}} t^{\beta_{1} \rho_{1}} \frac{\partial}{\partial s} f\left(t^{\rho} b^{\rho_{1}}+\left(1-t^{\rho_{1}}\right) a^{\rho_{1}}, s^{\rho_{2}} d^{\rho_{2}}+\left(1-s^{\rho_{2}}\right) c^{\rho_{2}}\right)\right|_{t=0} ^{t=1}\right. \\
& \left.-\frac{\beta_{1}}{\left(b^{\rho_{1}}-a^{\rho_{1}}\right)} \int_{0}^{1} t^{\beta_{1} \rho_{1}-1} \frac{\partial}{\partial s} f\left(t^{\rho_{1}} b^{\rho_{1}}+\left(1-t^{\rho_{1}}\right) a^{\rho_{1}}, s^{\rho_{2}} d^{\rho_{2}}+\left(1-s^{\rho_{2}}\right) c^{\rho_{2}}\right) d t\right] d s \\
& =\int_{0}^{1} s^{\left(\beta_{2}+1\right) \rho_{2}-1}\left[\frac{1}{\left(b^{\left.\rho_{1}-a^{\rho_{1}}\right) \rho_{1}}\right.} \frac{\partial}{\partial s} f\left(b^{\rho_{1}}, s^{\rho_{2}} d^{\rho_{2}}+\left(1-s^{\rho_{2}}\right) c^{\rho_{2}}\right)\right. \\
& \left.-\frac{\beta_{1}}{\left(b^{\rho_{1}}-a^{\rho_{1}}\right)} \int_{0}^{1} t^{\beta_{1} \rho_{1}-1} \frac{\partial}{\partial s} f\left(t^{\rho_{1}} b^{\rho_{1}}+\left(1-t^{\rho_{1}}\right) a^{\rho_{1}}, s^{\rho_{2}} d^{\rho_{2}}+\left(1-s^{\rho_{2}}\right) c^{\rho_{2}}\right) d t\right] d s \\
& =\frac{1}{\left(b^{\rho_{1}}-a^{\rho_{1}}\right) \rho_{1}} \int_{0}^{1} s^{\beta_{2} \rho_{2}} s^{\rho_{2}-1} \frac{\partial}{\partial s} f\left(b^{\rho_{1}}, s^{\rho_{2}} d^{\rho_{2}}+\left(1-s^{\rho_{2}}\right) c^{\rho_{2}}\right) d s-\frac{\beta_{1}}{\left(b^{\rho_{1}-a^{\rho_{1}}}\right)} \\
& \times \int_{0}^{1} t^{\beta_{1} \rho_{1}-1}\left[\int_{0}^{1} s^{\beta_{2} \rho_{2}} s^{\rho_{2}-1} \frac{\partial}{\partial s} f\left(t^{\rho_{1}} b^{\rho_{1}}+\left(1-t^{\rho_{1}}\right) a^{\rho_{1}}, s^{\rho_{2}} d^{\rho_{2}}+\left(1-s^{\rho_{2}}\right) c^{\rho_{2}}\right) d s\right] d t \\
& =\left.\frac{1}{\left(b^{\rho_{1}}-a^{\rho_{1}}\right)\left(y^{\left.\rho_{2}-c^{\rho_{2}}\right) \rho_{1} \rho_{2}}\right.} s^{\beta_{2} \rho_{2}} f\left(b^{\rho_{1}}, s^{\rho_{2}} d^{\rho_{2}}+\left(1-s^{\rho_{2}}\right) c^{\rho_{2}}\right)\right|_{s=0} ^{s=1} \\
& -\frac{\beta_{2}}{\left(b^{\rho_{1}}-a^{\rho_{1}}\right)\left(d^{\left.\rho_{2}-c^{\rho_{2}}\right) \rho_{1}}\right.} \int_{0}^{1} s^{\beta_{2} \rho_{2}-1} f\left(b^{\rho_{1}}, s^{\rho_{2}} d^{\rho_{2}}+\left(1-s^{\rho_{2}}\right) c^{\rho_{2}}\right) d s \\
& \frac{\beta_{1}}{\left(b^{\rho_{1}}-a^{\rho_{1}}\right)} \\
& \times \int_{0}^{1} t^{\beta_{1} \rho_{1}-1}\left[\left.\frac{1}{\left(d^{\left.\rho_{2}-c^{\rho_{2}}\right) \rho_{2}}\right.} s^{\beta_{2} \rho_{2}} f\left(t^{\rho_{1}} b^{\rho_{1}}+\left(1-t^{\rho_{1}}\right) a^{\rho_{1}}, s^{\rho_{2}} d^{\rho_{2}}+\left(1-s^{\rho_{2}}\right) c^{\rho_{2}}\right)\right|_{s=0} ^{s=1}\right. \\
& \left.-\frac{\beta_{2}}{\left(d^{\rho_{2}}-c^{\rho_{2}}\right)} \int_{0}^{1} s^{\beta_{2} \rho_{2}-1} f\left(t^{\rho_{1}} b^{\rho_{1}}+\left(1-t^{\rho_{1}}\right) a^{\rho_{1}}, s^{\rho_{2}} d^{\rho_{2}}+\left(1-s^{\rho_{2}}\right) c^{\rho_{2}}\right) d s\right] d t .
\end{aligned}
$$

Thus,

$$
\begin{aligned}
I_{1}= & \frac{1}{\left(b^{\rho_{1}}-a^{\rho_{1}}\right)\left(d^{\left.\rho_{2}-c^{\rho_{2}}\right) \rho_{1} \rho_{2}}\right.} f\left(b^{\rho_{1}}, d^{\rho_{2}}\right) \\
& -\frac{\beta_{2}}{\left(b^{\rho_{1}}-a^{\rho_{1}}\right)\left(d^{\left.\rho_{2}-c^{\rho_{2}}\right) \rho_{1}}\right.} \int_{0}^{1} s^{\beta_{2} \rho_{2}-1} f\left(b^{\rho_{1}}, s^{\rho_{2}} d^{\rho_{2}}+\left(1-s^{\rho_{2}}\right) c^{\rho_{2}}\right) d s \\
& -\frac{\beta_{1}}{\left(b^{\rho_{1}}-a^{\rho_{1}}\right)\left(d^{\left.\rho_{2}-c^{\rho_{2}}\right) \rho_{2}}\right.} \int_{0}^{1} t^{\beta_{1} \rho_{1}-1} f\left(t^{\rho_{1}} b^{\rho_{1}}+\left(1-t^{\rho_{1}}\right) a^{\rho_{1}}, d^{\rho_{2}}\right) d t \\
& +\frac{\beta_{1} \beta_{2}}{\left(b^{\rho_{1}}-a^{\rho_{1}}\right)\left(b^{\left.\rho_{2}-c^{\rho_{2}}\right)}\right.} \\
& \times \int_{0}^{1} \int_{0}^{1} t^{\beta_{1} \rho_{1}-1} s^{\beta_{2} \rho_{2}-1} f\left(t^{\rho_{1}} b^{\rho_{1}}+\left(1-t^{\rho_{1}}\right) a^{\rho_{1}}, s^{\rho_{2}} d^{\rho_{2}}+\left(1-s^{\rho_{2}}\right) c^{\rho_{2}}\right) d s d t
\end{aligned}
$$


By using Lemma 2.1, we have

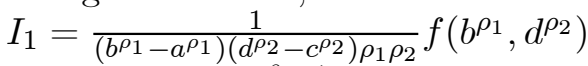

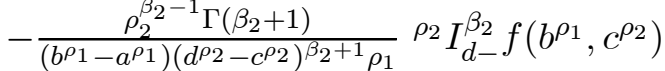

$$
\begin{aligned}
& -\frac{\rho_{1}^{\beta_{1}-1} \Gamma\left(\beta_{1}+1\right)}{\left(b^{\rho_{1}}-a^{\rho_{1}}\right)^{\beta_{1}+1}\left(d^{\rho_{2}}-c^{\rho_{2}}\right) \rho_{2}}{ }^{\rho_{1}} I_{b-}^{\beta_{1}} f\left(a^{\rho_{1}}, d^{\rho_{2}}\right) \\
& +\frac{\rho_{1}^{\beta_{1}-1} \rho_{2}^{\beta_{2}-1} \Gamma\left(\beta_{1}+1\right) \Gamma\left(\beta_{2}+1\right)}{\left(b^{\rho_{1}}-a^{\rho_{1}}\right)^{\beta_{1}+1}\left(d^{\left.\rho_{2}-c^{\rho_{2}}\right)^{\beta_{2}+1}} \rho_{1}, \rho_{2}\right.} I_{b-, d-}^{\beta_{1}, \beta_{2}} f\left(a^{\rho_{1}}, c^{\rho_{2}}\right) .
\end{aligned}
$$

So, it follows that

$$
\begin{aligned}
& \left(b^{\rho_{1}}-a^{\rho_{1}}\right)\left(d^{\rho_{2}}-c^{\rho_{2}}\right) \rho_{1} \rho_{2} I_{1} \\
& =f\left(b^{\rho_{1}}, d^{\rho_{2}}\right)-\frac{\rho_{2}^{\beta_{2}} \Gamma\left(\beta_{2}+1\right)}{\left(d^{\left.\rho_{2}-c^{\rho_{2}}\right)^{\beta_{2}}} \rho_{2}\right.} I_{d-}^{\beta_{2}} f\left(b^{\rho_{1}}, c^{\rho_{2}}\right)-\frac{\rho_{1}^{\beta_{1}} \Gamma\left(\beta_{1}+1\right)}{\left(b^{\rho_{1}}-a^{\rho_{1}}\right)^{\beta_{1}}} \rho_{1} I_{b-}^{\beta_{1}} f\left(a^{\rho_{1}}, d^{\rho_{2}}\right) \\
& \quad+\frac{\rho_{1}^{\beta_{1}} \rho_{2}^{\beta_{2}} \Gamma\left(\beta_{1}+1\right) \Gamma\left(\beta_{2}+1\right)}{\left(b^{\rho_{1}}-a^{\rho_{1}}\right)^{\beta_{1}}\left(d^{\left.\rho_{2}-c^{\rho_{2}}\right)^{\beta_{2}}}\right.} \rho_{1}, \rho_{2} I_{b-, d-}^{\beta_{1}, \beta_{2}} f\left(a^{\rho_{1}}, c^{\rho_{2}}\right) .
\end{aligned}
$$

By using similar arguments as in the above, we obtained the following.

$$
\begin{aligned}
& \left(b^{\rho_{1}}-a^{\rho_{1}}\right)\left(d^{\rho_{2}}-c^{\rho_{2}}\right) \rho_{1} \rho_{2} I_{2} \\
& =-f\left(b^{\rho_{1}}, c^{\rho_{2}}\right)+\frac{\rho_{2}^{\beta_{2}} \Gamma\left(\beta_{2}+1\right)}{\left(d^{\rho_{2}}-c^{\rho_{2}}\right)^{\beta_{2}}} \rho_{2} I_{c+}^{\beta_{2}} f\left(b^{\rho_{1}}, d^{\rho_{2}}\right)+\frac{\rho_{1}^{\beta_{1}} \Gamma\left(\beta_{1}+1\right)}{\left(b^{\rho_{1}}-a^{\rho_{1}}\right)^{\beta_{1}}} \rho_{1} I_{b-}^{\beta_{1}} f\left(a^{\rho_{1}}, c^{\rho_{2}}\right) \\
& -\frac{\rho_{1}^{\beta_{1}} \rho_{2}^{\beta_{2}} \Gamma\left(\beta_{1}+1\right) \Gamma\left(\beta_{2}+1\right)}{\left(b^{\rho_{1}}-a^{\rho_{1}}\right)^{\beta_{1}}\left(d^{\rho_{2}}-c^{\rho_{2}}\right)^{\beta_{2}}} \rho_{1}, \rho_{2} I_{b-, c+}^{\beta_{1}, \beta_{2}} f\left(a^{\rho_{1}}, d^{\rho_{2}}\right), \\
& \left(b^{\rho_{1}}-a^{\rho_{1}}\right)\left(d^{\rho_{2}}-c^{\rho_{2}}\right) \rho_{1} \rho_{2} I_{3} \\
& =-f\left(a^{\rho_{1}}, d^{\rho_{2}}\right)+\frac{\rho_{2}^{\beta_{2}} \Gamma\left(\beta_{2}+1\right)}{\left(d^{\rho_{2}}-c^{\rho_{2}}\right)^{\beta_{2}}} \rho_{2} I_{d-}^{\beta_{2}} f\left(a^{\rho_{1}}, c^{\rho_{2}}\right)+\frac{\rho_{1}^{\beta_{1}} \Gamma\left(\beta_{1}+1\right)}{\left(b^{\rho_{1}}-a^{\rho_{1}}\right)^{\beta_{1}}} \rho_{1} I_{a+}^{\beta_{1}} f\left(b^{\rho_{1}}, d^{\rho_{2}}\right)
\end{aligned}
$$

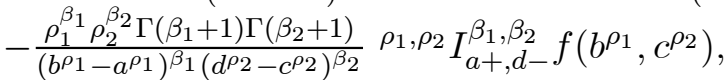

and

$$
\begin{aligned}
& \left(b^{\rho_{1}}-a^{\rho_{1}}\right)\left(d^{\rho_{2}}-c^{\rho_{2}}\right) \rho_{1} \rho_{2} I_{4} \\
& =f\left(a^{\rho_{1}}, c^{\rho_{2}}\right)-\frac{\rho_{2}^{\beta_{2}} \Gamma\left(\beta_{2}+1\right)}{\left(d^{\rho_{2}}-c^{\rho_{2}}\right)^{\beta_{2}}} \rho_{2} I_{c+}^{\beta_{2}} f\left(a^{\rho_{1}}, d^{\rho_{2}}\right)-\frac{\rho_{1}^{\beta_{1}} \Gamma\left(\beta_{1}+1\right)}{\left(b^{\rho_{1}}-a^{\rho_{1}}\right)^{\beta_{1}}} \rho_{1} I_{a+}^{\beta_{1}} f\left(b^{\rho_{1}}, c^{\rho_{2}}\right) \\
& \quad+\frac{\rho_{1}^{\beta_{1}} \rho_{2}^{\beta_{2}} \Gamma\left(\beta_{1}+1\right) \Gamma\left(\beta_{2}+1\right)}{\left(b^{\rho_{1}}-a^{\rho_{1}}\right)^{\beta_{1}}\left(d^{\left.\rho_{2}-c^{\rho_{2}}\right)^{\beta_{2}}}\right.} \rho_{1}, \rho_{2} I_{a+, c+}^{\beta_{1}, \beta_{2}} f\left(b^{\rho_{1}}, d^{\rho_{2}}\right) .
\end{aligned}
$$

The desired identity follows from adding (2.8), (2.9), (2.10) and (2.11). 
Theorem 2.5. Let $\beta_{1}, \beta_{2}, \rho_{1}, \rho_{2}>0$ and $f:[0, \infty) \times[0, \infty) \rightarrow \mathbf{R}$ be a twice partial differentiable mapping on $\left(a^{\rho_{1}}, b^{\rho_{1}}\right) \times\left(c^{\rho_{2}}, d^{\rho_{2}}\right)$ with $0 \leq a<b$ and $0 \leq c<d$, and $\frac{\partial^{2} f}{\partial t \partial s} \in L_{1}\left(\left[a^{\rho_{1}}, b^{\rho_{1}}\right] \times\left[c^{\rho_{2}}, d^{\rho_{2}}\right]\right)$. If $\left|\frac{\partial^{2} f}{\partial s \partial t}\right|^{q}$ is $(\alpha, m)$ convex on the coordinates on $\left(a^{\rho_{1}}, b^{\rho_{1}}\right) \times\left(c^{\rho_{2}}, d^{\rho_{2}}\right)$ for $(\alpha, m) \in(0,1] \times(0,1]$ and $q \geq 1$, then the following inequality holds:

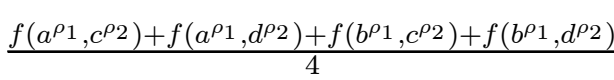

$$
\begin{aligned}
& -\frac{\rho_{2}^{\beta_{2}} \Gamma\left(\beta_{2}+1\right)}{4\left(d^{\left.\rho_{2}-c^{\rho_{2}}\right)^{\beta_{2}}}\right.}\left[{ }^{\rho_{2}} I_{c+}^{\beta_{2}} f\left(a^{\rho_{1}}, d^{\rho_{2}}\right)+{ }^{\rho_{2}} I_{c+}^{\beta_{2}} f\left(b^{\rho_{1}}, d^{\rho_{2}}\right)\right. \\
& \left.+{ }^{\rho_{2}} I_{d-}^{\beta_{2}} f\left(a^{\rho_{1}}, c^{\rho_{2}}\right)+{ }^{\rho_{2}} I_{d-}^{\beta_{2}} f\left(b^{\rho_{1}}, c^{\rho_{2}}\right)\right] \\
& -\frac{\rho_{1}^{\beta_{1}} \Gamma\left(\beta_{1}+1\right)}{4\left(b^{\rho_{1}}-a^{\rho_{1}}\right)^{\beta_{1}}}\left[{ }^{\rho_{1}} I_{a+}^{\beta_{1}} f\left(b^{\rho_{1}}, c^{\rho_{2}}\right)+{ }^{\rho_{1}} I_{a+}^{\beta_{1}} f\left(b^{\rho_{1}}, d^{\rho_{2}}\right)\right. \\
& \left.+{ }^{\rho_{1}} I_{b-}^{\beta_{1}} f\left(a^{\rho_{1}}, c^{\rho_{2}}\right)+{ }^{\rho_{1}} I_{b-}^{\beta_{1}} f\left(a^{\rho_{1}}, d^{\rho_{2}}\right)\right] \\
& +\frac{\rho_{1}^{\beta_{1}} \rho_{2}^{\beta_{2}} \Gamma\left(\beta_{1}+1\right) \Gamma\left(\beta_{2}+1\right)}{4\left(b^{\rho_{1}}-a^{\rho_{1}}\right)^{\beta_{1}}\left(d^{\rho_{2}}-c^{\rho_{2}}\right)^{\beta_{2}}}\left[{ }^{\rho_{1}, \rho_{2}} I_{a+, c+}^{\beta_{1}, \beta_{2}} f\left(b^{\rho_{1}}, d^{\rho_{2}}\right)+{ }^{\rho_{1}, \rho_{2}} I_{a+, d-}^{\beta_{1}, \beta_{2}} f\left(b^{\rho_{1}}, c^{\rho_{2}}\right)\right. \\
& \left.+^{\rho_{1}, \rho_{2}} I_{b-, c+}^{\beta_{1}, \beta_{2}} f\left(c^{\rho_{1}}, d^{\rho_{2}}\right)+{ }^{\rho_{1}, \rho_{2}} I_{b-, d-}^{\beta_{1}, \beta_{2}} f\left(a^{\rho_{1}}, c^{\rho_{2}}\right)\right] \\
& \leq \frac{\rho_{1} \rho_{2}\left(b^{\rho_{1}}-a^{\rho_{1}}\right)\left(d^{\rho_{2}}-c^{\rho_{2}}\right)}{4}\left(\frac{1}{\left(\beta_{1}+1\right)\left(\beta_{2}+1\right) \rho_{1} \rho_{2}}\right)^{1-\frac{1}{q}} \\
& \times\left\{\left(\frac{1}{\left(\beta_{1}+\alpha+1\right)\left(\beta_{2}+\alpha+1\right) \rho_{1} \rho_{2}}\left|\frac{\partial^{2}}{\partial t \partial s} f\left(b^{\rho_{1}}, d^{\rho_{2}}\right)\right|^{q}\right.\right. \\
& \begin{array}{l}
+\frac{1}{\left(\beta_{1}+\alpha+1\right) \rho_{1}}\left(\frac{1}{\left(\beta_{2}+1\right) \rho_{2}}-\frac{1}{\left(\beta_{2}+\alpha+1\right) \rho_{2}}\right) m\left|\frac{\partial^{2}}{\partial t \partial s} f\left(b^{\rho_{1}}, \frac{c^{\rho_{2}}}{m}\right)\right|^{q} \\
+\frac{1}{\left(\beta_{2}+\alpha+1\right) \rho_{2}}\left(\frac{1}{\left(\beta_{1}+1\right) \rho_{1}}-\frac{1}{\left(\beta_{1}+\alpha+1\right) \rho_{1}}\right) m\left|\frac{\partial^{2}}{\partial t \partial s} f\left(\frac{a^{\rho_{1}}}{m}, d^{\rho_{2}}\right)\right|^{q}
\end{array} \\
& +\left(\frac{1}{\left(\beta_{2}+1\right) \rho_{2}}-\frac{1}{\left(\beta_{2}+\alpha+1\right) \rho_{2}}\right)\left(\frac{1}{\left(\beta_{1}+1\right) \rho_{1}}-\frac{1}{\left(\beta_{1}+\alpha+1\right) \rho_{1}}\right) \\
& \left.\times m^{2}\left|\frac{\partial^{2}}{\partial t \partial s} f\left(\frac{a^{\rho_{1}}}{m}, \frac{c^{\rho_{2}}}{m}\right)\right|^{q}\right)^{\frac{1}{q}}
\end{aligned}
$$




$$
\begin{aligned}
& +\left(\frac{1}{\left(\beta_{1}+\alpha+1\right)\left(\beta_{2}+\alpha+1\right) \rho_{1} \rho_{2}}\left|\frac{\partial^{2}}{\partial t \partial s} f\left(b^{\rho_{1}}, c^{\rho_{2}}\right)\right|^{q}\right. \\
& +\frac{1}{\left(\beta_{1}+\alpha+1\right) \rho_{1}}\left(\frac{1}{\left(\beta_{2}+1\right) \rho_{2}}-\frac{1}{\left(\beta_{2}+\alpha+1\right) \rho_{2}}\right) m\left|\frac{\partial^{2}}{\partial t \partial s} f\left(b^{\rho_{1}}, \frac{d^{\rho_{2}}}{m}\right)\right|^{q} \\
& +\frac{1}{\left(\beta_{2}+\alpha+1\right) \rho_{2}}\left(\frac{1}{\left(\beta_{1}+1\right) \rho_{1}}-\frac{1}{\left(\beta_{1}+\alpha+1\right) \rho_{1}}\right) m\left|\frac{\partial^{2}}{\partial t \partial s} f\left(\frac{a^{\rho_{1}}}{m}, c^{\rho_{2}}\right)\right|^{q} \\
& +\left(\frac{1}{\left(\beta_{2}+1\right) \rho_{2}}-\frac{1}{\left(\beta_{2}+\alpha+1\right) \rho_{2}}\right)\left(\frac{1}{\left(\beta_{1}+1\right) \rho_{1}}-\frac{1}{\left(\beta_{1}+\alpha+1\right) \rho_{1}}\right) \\
& \left.\times m^{2}\left|\frac{\partial^{2}}{\partial t \partial s} f\left(\frac{a^{\rho_{1}}}{m}, \frac{d^{\rho_{2}}}{m}\right)\right|^{q}\right)^{\frac{1}{q}} \\
& +\left(\frac{1}{\left(\beta_{1}+\alpha+1\right)\left(\beta_{2}+\alpha+1\right) \rho_{1} \rho_{2}}\left|\frac{\partial^{2}}{\partial t \partial s} f\left(a^{\rho_{1}}, d^{\rho_{2}}\right)\right|^{q}\right. \\
& \begin{array}{l}
+\frac{1}{\left(\beta_{1}+\alpha+1\right) \rho_{1}}\left(\frac{1}{\left(\beta_{2}+1\right) \rho_{2}}-\frac{1}{\left(\beta_{2}+\alpha+1\right) \rho_{2}}\right) m\left|\frac{\partial^{2}}{\partial t \partial s} f\left(a^{\rho_{1}}, \frac{c^{\rho_{2}}}{m}\right)\right|^{q} \\
+\frac{1}{\left(\beta_{2}+\alpha+1\right) \rho_{2}}\left(\frac{1}{\left(\beta_{1}+1\right) \rho_{1}}-\frac{1}{\left(\beta_{1}+\alpha+1\right) \rho_{1}}\right) m\left|\frac{\partial^{2}}{\partial t \partial s} f\left(\frac{b^{\rho_{1}}}{m}, d^{\rho_{2}}\right)\right|^{q}
\end{array} \\
& +\left(\frac{1}{\left(\beta_{2}+1\right) \rho_{2}}-\frac{1}{\left(\beta_{2}+\alpha+1\right) \rho_{2}}\right)\left(\frac{1}{\left(\beta_{1}+1\right) \rho_{1}}-\frac{1}{\left(\beta_{1}+\alpha+1\right) \rho_{1}}\right) \\
& \left.\times m^{2}\left|\frac{\partial^{2}}{\partial t \partial s} f\left(\frac{b^{\rho_{1}}}{m}, \frac{c^{\rho_{2}}}{m}\right)\right|^{q}\right)^{\frac{1}{q}} \\
& +\left(\frac{1}{\left(\beta_{1}+\alpha+1\right)\left(\beta_{2}+\alpha+1\right) \rho_{1} \rho_{2}}\left|\frac{\partial^{2}}{\partial t \partial s} f\left(a^{\rho_{1}}, c^{\rho_{2}}\right)\right|^{q}\right. \\
& +\frac{1}{\left(\beta_{1}+\alpha+1\right) \rho_{1}}\left(\frac{1}{\left(\beta_{2}+1\right) \rho_{2}}-\frac{1}{\left(\beta_{2}+\alpha+1\right) \rho_{2}}\right) m\left|\frac{\partial^{2}}{\partial t \partial s} f\left(a^{\rho_{1}}, \frac{d^{\rho_{2}}}{m}\right)\right|^{q} \\
& +\frac{1}{\left(\beta_{2}+\alpha+1\right) \rho_{2}}\left(\frac{1}{\left(\beta_{1}+1\right) \rho_{1}}-\frac{1}{\left(\beta_{1}+\alpha+1\right) \rho_{1}}\right) m\left|\frac{\partial^{2}}{\partial t \partial s} f\left(\frac{b^{\rho_{1}}}{m}, c^{\rho_{2}}\right)\right|^{q} \\
& +\left(\frac{1}{\left(\beta_{2}+1\right) \rho_{2}}-\frac{1}{\left(\beta_{2}+\alpha+1\right) \rho_{2}}\right)\left(\frac{1}{\left(\beta_{1}+1\right) \rho_{1}}-\frac{1}{\left(\beta_{1}+\alpha+1\right) \rho_{1}}\right) \\
& \left.\left.\times m^{2}\left|\frac{\partial^{2}}{\partial t \partial s} f\left(\frac{b^{\rho_{1}}}{m}, \frac{d^{\rho_{2}}}{m}\right)\right|^{q}\right)^{\frac{1}{q}}\right\} \text {. }
\end{aligned}
$$

Proof. By using Lemma 2.4 and the properties of the absolute value, we obtain 


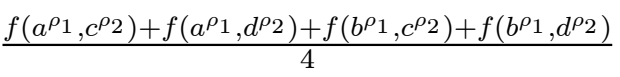

$$
\begin{aligned}
& -\frac{\rho_{2}^{\beta_{2}} \Gamma\left(\beta_{2}+1\right)}{4\left(d^{\rho_{2}}-c^{\rho_{2}}\right)^{\beta_{2}}}\left[\rho^{\rho_{2}} I_{c+}^{\beta_{2}} f\left(a^{\rho_{1}}, d^{\rho_{2}}\right)+{ }^{\rho_{2}} I_{c+}^{\beta_{2}} f\left(b^{\rho_{1}}, d^{\rho_{2}}\right)\right. \\
& \left.+{ }^{\rho_{2}} I_{d-}^{\beta_{2}} f\left(a^{\rho_{1}}, c^{\rho_{2}}\right)+{ }^{\rho_{2}} I_{d-}^{\beta_{2}} f\left(b^{\rho_{1}}, c^{\rho_{2}}\right)\right] \\
& -\frac{\rho_{1}^{\beta_{1}} \Gamma\left(\beta_{1}+1\right)}{4\left(b^{\rho_{1}}-a^{\rho_{1}}\right)^{\beta_{1}}}\left[{ }^{\rho_{1}} I_{a+}^{\beta_{1}} f\left(b^{\rho_{1}}, c^{\rho_{2}}\right)+{ }^{\rho_{1}} I_{a+}^{\beta_{1}} f\left(b^{\rho_{1}}, d^{\rho_{2}}\right)\right. \\
& \left.+{ }^{\rho_{1}} I_{b-}^{\beta_{1}} f\left(a^{\rho_{1}}, c^{\rho_{2}}\right)+{ }^{\rho_{1}} I_{b-}^{\beta_{1}} f\left(a^{\rho_{1}}, d^{\rho_{2}}\right)\right] \\
& +\frac{\rho_{1}^{\beta_{1}} \rho_{2}^{\beta_{2}} \Gamma\left(\beta_{1}+1\right) \Gamma\left(\beta_{2}+1\right)}{4\left(b^{\rho_{1}}-a^{\rho_{1}}\right)^{\beta_{1}}\left(d^{\rho_{2}}-c^{\rho_{2}}\right)^{\beta_{2}}}\left[{ }^{\rho_{1}, \rho_{2}} I_{a+, c+}^{\beta_{1}, \beta_{2}} f\left(b^{\rho_{1}}, d^{\rho_{2}}\right)+{ }^{\rho_{1}, \rho_{2}} I_{a+, d-}^{\beta_{1}, \beta_{2}} f\left(b^{\rho_{1}}, c^{\rho_{2}}\right)\right. \\
& \left.+{ }^{\rho_{1}, \rho_{2}} I_{b-, c+}^{\beta_{1}, \beta_{2}} f\left(c^{\rho_{1}}, d^{\rho_{2}}\right)+{ }^{\rho_{1}, \rho_{2}} I_{b-, d-}^{\beta_{1}, \beta_{2}} f\left(a^{\rho_{1}}, c^{\rho_{2}}\right)\right] \mid \\
& \leq \frac{\rho_{1} \rho_{2}\left(b^{\rho_{1}}-a^{\rho_{1}}\right)\left(d^{\rho_{2}}-c^{\rho_{2}}\right)}{4}\left(\left|I_{1}\right|+\left|I_{2}\right|+\left|I_{3}\right|+I_{4} \mid\right) \text {. }
\end{aligned}
$$

By using the power mean inequality and the $(\alpha, m)$-convexity of $\left|\frac{\partial^{2} f}{\partial t \partial s}\right|^{q}$ on the coordinates, we have

$$
\begin{aligned}
\left|I_{1}\right| \leq & \left(\int_{0}^{1} \int_{0}^{1} s^{\left(\beta_{2}+1\right) \rho_{2}-1} t^{\left(\beta_{1}+1\right) \rho_{1}-1} d s d t\right)^{1-\frac{1}{q}}\left(\int_{0}^{1} \int_{0}^{1} s^{\left(\beta_{2}+1\right) \rho_{2}-1} t^{\left(\beta_{1}+1\right) \rho_{1}-1}\right. \\
& \left.\times\left|\frac{\partial^{2}}{\partial t \partial s} f\left(t^{\rho_{1}} b^{\rho_{1}}+\left(1-t^{\rho_{1}}\right) a^{\rho_{1}}, s^{\rho_{2}} d^{\rho_{2}}+\left(1-s^{\rho_{2}}\right) c^{\rho_{2}}\right)\right|^{q} d t d s\right)^{\frac{1}{q}} \\
\leq & \left(\frac{1}{\left(\beta_{1}+1\right)\left(\beta_{2}+1\right) \rho_{1} \rho_{2}}\right) \\
& \times\left(\left|\frac{\partial^{2}}{\partial t \partial s} f\left(b^{\rho_{1}}, d^{\rho_{2}}\right)\right| \int_{0}^{1} \int_{0}^{1} s^{\left(\beta_{2}+1\right) \rho_{2}-1} t^{\left(\beta_{1}+1\right) \rho_{1}-1} s^{\alpha \rho_{2}} t^{\alpha \rho_{1}} d t d s\right. \\
& +m\left|\frac{\partial^{2}}{\partial t \partial s} f\left(b^{\rho_{1}}, \frac{c^{\rho_{2}}}{m}\right)\right|^{q} \int_{0}^{1} \int_{0}^{1} s^{\left(\beta_{2}+1\right) \rho_{2}-1} t^{\left(\beta_{1}+1\right) \rho_{1}-1}\left(1-s^{\alpha \rho_{2}}\right) t^{\alpha \rho_{1}} d t d s \\
& +m\left|\frac{\partial^{2}}{\partial t \partial s} f\left(\frac{a^{\rho_{1}}}{m}, d^{\rho_{2}}\right)\right|^{q} \int_{0}^{1} \int_{0}^{1} s^{\left(\beta_{2}+1\right) \rho_{2}-1} t^{\left(\beta_{1}+1\right) \rho_{1}-1} s^{\alpha \rho_{2}}\left(1-t^{\alpha \rho_{1}}\right) d t d s \\
& \left.+m^{2}\left|\frac{\partial^{2}}{\partial t \partial s} f\left(\frac{a^{\rho_{1}}}{m}, \frac{c^{\rho_{2}}}{m}\right)\right|^{q} \int_{0}^{1} \int_{0}^{1} s^{\left(\beta_{2}+1\right) \rho_{2}-1} t^{\left(\beta_{1}+1\right) \rho_{1}-1}\left(1-s^{\alpha \rho_{2}}\right)\left(1-t^{\alpha \rho_{1}}\right) d t d s\right)^{\frac{1}{q}} .
\end{aligned}
$$


Thus,

$$
\begin{aligned}
\left|I_{1}\right| \leq & \left(\frac{1}{\left(\beta_{1}+1\right)\left(\beta_{2}+1\right) \rho_{1} \rho_{2}}\right)^{1-\frac{1}{q}}\left(\frac{1}{\left(\beta_{1}+\alpha+1\right)\left(\beta_{2}+\alpha+1\right) \rho_{1} \rho_{2}}\left|\frac{\partial^{2}}{\partial t \partial s} f\left(b^{\rho_{1}}, d^{\rho_{2}}\right)\right|^{q}\right. \\
& +\frac{1}{\left(\beta_{1}+\alpha+1\right) \rho_{1}}\left(\frac{1}{\left(\beta_{2}+1\right) \rho_{2}}-\frac{1}{\left(\beta_{2}+\alpha+1\right) \rho_{2}}\right) m\left|\frac{\partial^{2}}{\partial t \partial s} f\left(b^{\rho_{1}}, \frac{c^{\rho_{2}}}{m}\right)\right|^{q} \\
& +\frac{1}{\left(\beta_{2}+\alpha+1\right) \rho_{2}}\left(\frac{1}{\left(\beta_{1}+1\right) \rho_{1}}-\frac{1}{\left(\beta_{1}+\alpha+1\right) \rho_{1}}\right) m\left|\frac{\partial^{2}}{\partial t \partial s} f\left(\frac{a^{\rho_{1}}}{m}, d^{\rho_{2}}\right)\right|^{q} \\
& +\left(\frac{1}{\left(\beta_{2}+1\right) \rho_{2}}-\frac{1}{\left(\beta_{2}+\alpha+1\right) \rho_{2}}\right)\left(\frac{1}{\left(\beta_{1}+1\right) \rho_{1}}-\frac{1}{\left(\beta_{1}+\alpha+1\right) \rho_{1}}\right) \\
& \left.\times m^{2}\left|\frac{\partial^{2}}{\partial t \partial s} f\left(\frac{a^{\rho_{1}}}{m}, \frac{c^{\rho_{2}}}{m}\right)\right|^{q}\right)^{\frac{1}{q}} .
\end{aligned}
$$

Using similar argument, we have

$$
\begin{aligned}
\left|I_{2}\right| \leq & \left(\frac{1}{\left(\beta_{1}+1\right)\left(\beta_{2}+1\right) \rho_{1} \rho_{2}}\right)^{1-\frac{1}{q}}\left(\frac{1}{\left(\beta_{1}+\alpha+1\right)\left(\beta_{2}+\alpha+1\right) \rho_{1} \rho_{2}}\left|\frac{\partial^{2}}{\partial t \partial s} f\left(b^{\rho_{1}}, c^{\rho_{2}}\right)\right|^{q}\right. \\
& +\frac{1}{\left(\beta_{1}+\alpha+1\right) \rho_{1}}\left(\frac{1}{\left(\beta_{2}+1\right) \rho_{2}}-\frac{1}{\left(\beta_{2}+\alpha+1\right) \rho_{2}}\right) m\left|\frac{\partial^{2}}{\partial t \partial s} f\left(b^{\rho_{1}}, \frac{d^{\rho_{2}}}{m}\right)\right|^{q} \\
& +\frac{1}{\left(\beta_{2}+\alpha+1\right) \rho_{2}}\left(\frac{1}{\left(\beta_{1}+1\right) \rho_{1}}-\frac{1}{\left(\beta_{1}+\alpha+1\right) \rho_{1}}\right) m\left|\frac{\partial^{2}}{\partial t \partial s} f\left(\frac{a^{\rho_{1}}}{m}, c^{\rho_{2}}\right)\right|^{q} \\
& +\left(\frac{1}{\left(\beta_{2}+1\right) \rho_{2}}-\frac{1}{\left(\beta_{2}+\alpha+1\right) \rho_{2}}\right)\left(\frac{1}{\left(\beta_{1}+1\right) \rho_{1}}-\frac{1}{\left(\beta_{1}+\alpha+1\right) \rho_{1}}\right) \\
& \left.\times m^{2}\left|\frac{\partial^{2}}{\partial t \partial s} f\left(\frac{a^{\rho_{1}}}{m}, \frac{d^{\rho_{2}}}{m}\right)\right|^{q}\right)^{\frac{1}{q}}
\end{aligned}
$$$$
\left|I_{3}\right| \leq\left(\frac{1}{\left(\beta_{1}+1\right)\left(\beta_{2}+1\right) \rho_{1} \rho_{2}}\right)^{1-\frac{1}{q}}\left(\frac{1}{\left(\beta_{1}+\alpha+1\right)\left(\beta_{2}+\alpha+1\right) \rho_{1} \rho_{2}}\left|\frac{\partial^{2}}{\partial t \partial s} f\left(a^{\rho_{1}}, d^{\rho_{2}}\right)\right|^{q}\right.
$$

$$
\begin{aligned}
& +\frac{1}{\left(\beta_{1}+\alpha+1\right) \rho_{1}}\left(\frac{1}{\left(\beta_{2}+1\right) \rho_{2}}-\frac{1}{\left(\beta_{2}+\alpha+1\right) \rho_{2}}\right) m\left|\frac{\partial^{2}}{\partial t \partial s} f\left(a^{\rho_{1}}, \frac{c^{\rho_{2}}}{m}\right)\right|^{q} \\
& +\frac{1}{\left(\beta_{2}+\alpha+1\right) \rho_{2}}\left(\frac{1}{\left(\beta_{1}+1\right) \rho_{1}}-\frac{1}{\left(\beta_{1}+\alpha+1\right) \rho_{1}}\right) m\left|\frac{\partial^{2}}{\partial t \partial s} f\left(\frac{b^{\rho_{1}}}{m}, d^{\rho_{2}}\right)\right|^{q} \\
& +\left(\frac{1}{\left(\beta_{2}+1\right) \rho_{2}}-\frac{1}{\left(\beta_{2}+\alpha+1\right) \rho_{2}}\right)\left(\frac{1}{\left(\beta_{1}+1\right) \rho_{1}}-\frac{1}{\left(\beta_{1}+\alpha+1\right) \rho_{1}}\right) \\
& \left.\times m^{2}\left|\frac{\partial^{2}}{\partial t \partial s} f\left(\frac{b^{\rho_{1}}}{m}, \frac{\rho^{\rho} 2}{m}\right)\right|^{q}\right)^{\frac{1}{q}}
\end{aligned}
$$


and

$$
\begin{aligned}
\left|I_{4}\right| \leq & \left(\frac{1}{\left(\beta_{1}+1\right)\left(\beta_{2}+1\right) \rho_{1} \rho_{2}}\right)^{1-\frac{1}{q}}\left(\frac{1}{\left(\beta_{1}+\alpha+1\right)\left(\beta_{2}+\alpha+1\right) \rho_{1} \rho_{2}}\left|\frac{\partial^{2}}{\partial t \partial s} f\left(a^{\rho_{1}}, c^{\rho_{2}}\right)\right|^{q}\right. \\
& +\frac{1}{\left(\beta_{1}+\alpha+1\right) \rho_{1}}\left(\frac{1}{\left(\beta_{2}+1\right) \rho_{2}}-\frac{1}{\left(\beta_{2}+\alpha+1\right) \rho_{2}}\right) m\left|\frac{\partial^{2}}{\partial t \partial s} f\left(a^{\rho_{1}}, \frac{d^{\rho_{2}}}{m}\right)\right|^{q} \\
& +\frac{1}{\left(\beta_{2}+\alpha+1\right) \rho_{2}}\left(\frac{1}{\left(\beta_{1}+1\right) \rho_{1}}-\frac{1}{\left(\beta_{1}+\alpha+1\right) \rho_{1}}\right) m\left|\frac{\partial^{2}}{\partial t \partial s} f\left(\frac{b^{\rho_{1}}}{m}, c^{\rho_{2}}\right)\right|^{q} \\
& +\left(\frac{1}{\left(\beta_{2}+1\right) \rho_{2}}-\frac{1}{\left(\beta_{2}+\alpha+1\right) \rho_{2}}\right)\left(\frac{1}{\left(\beta_{1}+1\right) \rho_{1}}-\frac{1}{\left(\beta_{1}+\alpha+1\right) \rho_{1}}\right) \\
& \left.\times m^{2}\left|\frac{\partial^{2}}{\partial t \partial s} f\left(\frac{b^{\rho_{1}}}{m}, \frac{d^{\rho_{2}}}{m}\right)\right|^{q}\right)^{\frac{1}{q}} .
\end{aligned}
$$

The desired inequality follows from (2.12) and using (2.13)-(2.16).

Corollary 2.6. Let $\beta_{1}, \beta_{2}, \rho_{1}, \rho_{2}>0$ and $f:[0, \infty) \times[0, \infty) \rightarrow \mathbf{R}$ be a twice partial differentiable mapping on $\left(a^{\rho_{1}}, b^{\rho_{1}}\right) \times\left(c^{\rho_{2}}, d^{\rho_{2}}\right)$ with $0 \leq a<b$ and $0 \leq c<d$, and $\frac{\partial^{2} f}{\partial t \partial s} \in L_{1}\left(\left[a^{\rho_{1}}, b^{\rho_{1}}\right] \times\left[c^{\rho_{2}}, d^{\rho_{2}}\right]\right)$. If $\left|\frac{\partial^{2} f}{\partial t \partial s}\right|$ is $(\alpha, m)$ convex on the coordinates on $\left(a^{\rho_{1}}, b^{\rho_{1}}\right) \times\left(c^{\rho_{2}}, d^{\rho_{2}}\right)$ for $(\alpha, m) \in(0,1] \times(0,1]$, then the following inequality holds:

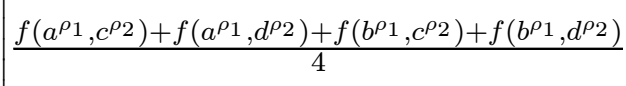

$$
\begin{aligned}
& -\frac{\rho_{2}^{\beta_{2}} \Gamma\left(\beta_{2}+1\right)}{4\left(d^{\rho_{2}}-c^{\rho_{2}}\right)^{\beta_{2}}}\left[\rho_{2} I_{c+}^{\beta_{2}} f\left(a^{\rho_{1}}, d^{\rho_{2}}\right)+{ }^{\rho_{2}} I_{c+}^{\beta_{2}} f\left(b^{\rho_{1}}, d^{\rho_{2}}\right)\right. \\
& \left.+{ }^{\rho_{2}} I_{d-}^{\beta_{2}} f\left(a^{\rho_{1}}, c^{\rho_{2}}\right)+{ }^{\rho_{2}} I_{d-}^{\beta_{2}} f\left(b^{\rho_{1}}, c^{\rho_{2}}\right)\right] \\
& -\frac{\rho_{1}^{\beta_{1}} \Gamma\left(\beta_{1}+1\right)}{4\left(b^{\rho_{1}}-a^{\rho_{1}}\right)^{\beta_{1}}}\left[{ }^{\rho_{1}} I_{a+}^{\beta_{1}} f\left(b^{\rho_{1}}, c^{\rho_{2}}\right)+{ }^{\rho_{1}} I_{a+}^{\beta_{1}} f\left(b^{\rho_{1}}, d^{\rho_{2}}\right)\right. \\
& \left.+{ }^{\rho_{1}} I_{b-}^{\beta_{1}} f\left(a^{\rho_{1}}, c^{\rho_{2}}\right)+{ }^{\rho_{1}} I_{b-}^{\beta_{1}} f\left(a^{\rho_{1}}, d^{\rho_{2}}\right)\right] \\
& +\frac{\rho_{1}^{\beta_{1}} \rho_{2}^{\beta_{2}} \Gamma\left(\beta_{1}+1\right) \Gamma\left(\beta_{2}+1\right)}{4\left(b^{\rho_{1}}-a^{\rho_{1}}\right)^{\beta_{1}}\left(d^{\left.\rho_{2}-c^{\rho_{2}}\right)^{\beta_{2}}}\right.}\left[{ }^{\rho_{1}, \rho_{2}} I_{a+, c+}^{\beta_{1}, \beta_{2}} f\left(b^{\rho_{1}}, d^{\rho_{2}}\right)+{ }^{\rho_{1}, \rho_{2}} I_{a+, d-}^{\beta_{1}, \beta_{2}} f\left(b^{\rho_{1}}, c^{\rho_{2}}\right)\right. \\
& \left.+^{\rho_{1}, \rho_{2}} I_{b-, c+}^{\beta_{1}, \beta_{2}} f\left(c^{\rho_{1}}, d^{\rho_{2}}\right)+{ }^{\rho_{1}, \rho_{2}} I_{b-, d-}^{\beta_{1}, \beta_{2}} f\left(a^{\rho_{1}}, c^{\rho_{2}}\right)\right] \\
& \leq \frac{\rho_{1} \rho_{2}\left(b^{\rho_{1}}-a^{\rho_{1}}\right)\left(d^{\rho_{2}}-c^{\rho_{2}}\right)}{4}
\end{aligned}
$$




$$
\begin{aligned}
& \times\left[\frac { 1 } { ( \beta _ { 1 } + \alpha + 1 ) ( \beta _ { 2 } + \alpha + 1 ) \rho _ { 1 } \rho _ { 2 } } \left(\left|\frac{\partial^{2}}{\partial t \partial s} f\left(b^{\rho_{1}}, d^{\rho_{2}}\right)\right|+\left|\frac{\partial^{2}}{\partial t \partial s} f\left(b^{\rho_{1}}, c^{\rho_{2}}\right)\right|\right.\right. \\
& \left.+\left|\frac{\partial^{2}}{\partial t \partial s} f\left(a^{\rho_{1}}, d^{\rho_{2}}\right)\right|+\left|\frac{\partial^{2}}{\partial t \partial s} f\left(a^{\rho_{1}}, c^{\rho_{2}}\right)\right|\right) \\
& +\frac{1}{\left(\beta_{1}+\alpha+1\right) \rho_{1}}\left(\frac{1}{\left(\beta_{2}+1\right) \rho_{2}}-\frac{1}{\left(\beta_{2}+\alpha+1\right) \rho_{2}}\right)\left(m\left|\frac{\partial^{2}}{\partial t \partial s} f\left(b^{\rho_{1}}, \frac{c^{\rho_{2}}}{m}\right)\right|\right. \\
& \left.+m\left|\frac{\partial^{2}}{\partial t \partial s} f\left(b^{\rho_{1}}, \frac{d^{\rho_{2}}}{m}\right)\right|+m\left|\frac{\partial^{2}}{\partial t \partial s} f\left(a^{\rho_{1}}, \frac{c^{\rho_{2}}}{m}\right)\right|+m\left|\frac{\partial^{2}}{\partial t \partial s} f\left(a^{\rho_{1}}, \frac{d^{\rho_{2}}}{m}\right)\right|\right) \\
& +\frac{1}{\left(\beta_{2}+\alpha+1\right) \rho_{2}}\left(\frac{1}{\left(\beta_{1}+1\right) \rho_{1}}-\frac{1}{\left(\beta_{1}+\alpha+1\right) \rho_{1}}\right)\left(m\left|\frac{\partial^{2}}{\partial t \partial s} f\left(\frac{a^{\rho_{1}}}{m}, d^{\rho_{2}}\right)\right|\right. \\
& +\left(\frac{1}{\left(\beta_{2}+1\right) \rho_{2}}-\frac{1}{\left(\beta_{2}+\alpha+1\right) \rho_{2}}\right)\left(\frac{1}{\left(\beta_{1}+1\right) \rho_{1}}-\frac{1}{\left(\beta_{1}+\alpha+1\right) \rho_{1}}\right) \\
& \times\left(m^{2}\left|\frac{\partial^{2}}{\partial t \partial s} f\left(\frac{a^{\rho_{1}}}{m}, \frac{c^{\rho_{2}}}{m}\right)\right|+m^{2}\left|\frac{\partial^{2}}{\partial t \partial s} f\left(\frac{a^{\rho_{1}}}{m}, \frac{d^{\rho_{2}}}{m}\right)\right|\right. \\
& \left.\left.\left.\quad+m^{2}\left|\frac{\partial^{2}}{\partial t \partial s} f\left(\frac{b^{\rho_{1}}}{m}, \frac{c^{\rho_{2}}}{m}\right)\right|+c^{\rho_{2}}\right)\left|\frac{\partial^{2}}{\partial t \partial s} f\left(\frac{b^{\rho_{1}}}{m}, \frac{d^{\rho_{2}}}{m}\right)\right|\right)\right]
\end{aligned}
$$

Proof. The result follows directly from Theorem 2.5, if we take $q=1$.

Theorem 2.7. Let $\beta_{1}, \beta_{2}, \rho_{1}, \rho_{2}>0$ and $f:[0, \infty) \times[0, \infty) \rightarrow \mathbf{R}$ be a twice partial differentiable mapping on $\left(a^{\rho_{1}}, b^{\rho_{1}}\right) \times\left(c^{\rho_{2}}, d^{\rho_{2}}\right)$ with $0 \leq a<b$ and $0 \leq c<d$, and $\frac{\partial^{2} f}{\partial t \partial s} \in L_{1}\left(\left[a^{\rho_{1}}, b^{\rho_{1}}\right] \times\left[c^{\rho_{2}}, d^{\rho_{2}}\right]\right)$. If $\left|\frac{\partial^{2} f}{\partial t \partial s}\right|^{q}$ is $(\alpha, m)$ convex on the coordinates on $\left(a^{\rho_{1}}, b^{\rho_{1}}\right) \times\left(c^{\rho_{2}}, d^{\rho_{2}}\right)$ for $(\alpha, m) \in(0,1] \times(0,1]$ and $q>1$, then the following inequality holds: 


$$
\begin{aligned}
& \frac{f\left(a^{\rho_{1}}, c^{\rho_{2}}\right)+f\left(a^{\rho_{1}}, d^{\rho_{2}}\right)+f\left(b^{\rho_{1}}, c^{\rho_{2}}\right)+f\left(b^{\left.\rho_{1}, d^{\rho_{2}}\right)}\right.}{4} \\
& -\frac{\rho_{2}^{\beta_{2}} \Gamma\left(\beta_{2}+1\right)}{4\left(d^{\left.\rho_{2}-c^{\rho_{2}}\right)^{\beta_{2}}}\right.}\left[\rho_{2} I_{c+}^{\beta_{2}} f\left(a^{\rho_{1}}, d^{\rho_{2}}\right)+{ }^{\rho_{2}} I_{c+}^{\beta_{2}} f\left(b^{\rho_{1}}, d^{\rho_{2}}\right)\right. \\
& \left.+{ }^{\rho_{2}} I_{d-}^{\beta_{2}} f\left(a^{\rho_{1}}, c^{\rho_{2}}\right)+{ }^{\rho_{2}} I_{d-}^{\beta_{2}} f\left(b^{\rho_{1}}, c^{\rho_{2}}\right)\right] \\
& -\frac{\rho_{1}^{\beta_{1}} \Gamma\left(\beta_{1}+1\right)}{4\left(b^{\rho_{1}}-a^{\rho_{1}}\right)^{\beta_{1}}}\left[\rho_{1} I_{a+}^{\beta_{1}} f\left(b^{\rho_{1}}, c^{\rho_{2}}\right)+{ }^{\rho_{1}} I_{a+}^{\beta_{1}} f\left(b^{\rho_{1}}, d^{\rho_{2}}\right)\right. \\
& \left.+{ }^{\rho_{1}} I_{b-}^{\beta_{1}} f\left(a^{\rho_{1}}, c^{\rho_{2}}\right)+{ }^{\rho_{1}} I_{b-}^{\beta_{1}} f\left(a^{\rho_{1}}, d^{\rho_{2}}\right)\right] \\
& +\frac{\rho_{1}^{\beta_{1}} \rho_{2}^{\beta_{2}} \Gamma\left(\beta_{1}+1\right) \Gamma\left(\beta_{2}+1\right)}{4\left(b^{\rho_{1}}-a^{\rho_{1}}\right)^{\beta_{1}}\left(d^{\rho_{2}}-c^{\rho_{2}}\right)^{\beta_{2}}}\left[\rho^{\rho_{1}, \rho_{2}} I_{a+, c+}^{\beta_{1}, \beta_{2}} f\left(b^{\rho_{1}}, d^{\rho_{2}}\right)+{ }^{\rho_{1}, \rho_{2}} I_{a+, d-}^{\beta_{1}, \beta_{2}} f\left(b^{\rho_{1}}, c^{\rho_{2}}\right)\right. \\
& \left.+^{\rho_{1}, \rho_{2}} I_{b-, c+}^{\beta_{1}, \beta_{2}} f\left(c^{\rho_{1}}, d^{\rho_{2}}\right)+{ }^{\rho_{1}, \rho_{2}} I_{b-, d-}^{\beta_{1}, \beta_{2}} f\left(a^{\rho_{1}}, c^{\rho_{2}}\right)\right] \\
& \leq \frac{\rho_{1} \rho_{2}\left(b^{\rho_{1}}-a^{\rho_{1}}\right)\left(d^{\left.\rho_{2}-c^{\rho_{2}}\right)}\right.}{4}\left(\frac{1}{\left(\left(\beta_{1}+1\right) \rho_{1} r-r+1\right)\left(\left(\beta_{2}+1\right) \rho_{2} r-r+1\right)}\right)^{\frac{1}{r}} \\
& \times\left(\frac{1}{\left(\alpha \rho_{1}+1\right)\left(\alpha \rho_{2}+1\right)}\right)^{\frac{1}{q}}\left\{\left(\left|\frac{\partial^{2}}{\partial t \partial s} f\left(b^{\rho_{1}}, d^{\rho_{2}}\right)\right|^{q}+\alpha \rho_{2} m\left|\frac{\partial^{2}}{\partial t \partial s} f\left(b^{\rho_{1}}, \frac{c^{\rho_{2}}}{m}\right)\right|^{q}\right.\right. \\
& \left.+\alpha \rho_{1} m\left|\frac{\partial^{2}}{\partial t \partial s} f\left(\frac{a^{\rho_{1}}}{m}, d^{\rho_{2}}\right)\right|^{q}+\alpha^{2} m^{2} \rho_{1} \rho_{2}\left|\frac{\partial^{2}}{\partial t \partial s} f\left(\frac{a^{\rho_{1}}}{m}, \frac{c^{\rho_{2}}}{m}\right)\right|^{q}\right)^{\frac{1}{q}} \\
& +\left(\left|\frac{\partial^{2}}{\partial t \partial s} f\left(b^{\rho_{1}}, c^{\rho_{2}}\right)\right|^{q}+\alpha \rho_{2} m\left|\frac{\partial^{2}}{\partial t \partial s} f\left(b^{\rho_{1}}, \frac{d^{\rho_{2}}}{m}\right)\right|^{q}\right. \\
& \left.+\alpha \rho_{1} m\left|\frac{\partial^{2}}{\partial t \partial s} f\left(\frac{a^{\rho_{1}}}{m}, c^{\rho_{2}}\right)\right|^{q}+\alpha^{2} m^{2} \rho_{1} \rho_{2}\left|\frac{\partial^{2}}{\partial t \partial s} f\left(\frac{a^{\rho_{1}}}{m}, \frac{d^{\rho_{2}}}{m}\right)\right|^{q}\right)^{\frac{1}{q}} \\
& +\left(\left|\frac{\partial^{2}}{\partial t \partial s} f\left(a^{\rho_{1}}, d^{\rho_{2}}\right)\right|^{q}+\alpha \rho_{2} m\left|\frac{\partial^{2}}{\partial t \partial s} f\left(a^{\rho_{1}}, \frac{c^{\rho_{2}}}{m}\right)\right|^{q}\right. \\
& \left.+\alpha \rho_{1} m\left|\frac{\partial^{2}}{\partial t \partial s} f\left(\frac{b^{\rho_{1}}}{m}, d^{\rho_{2}}\right)\right|^{q}+\alpha^{2} m^{2} \rho_{1} \rho_{2}\left|\frac{\partial^{2}}{\partial t \partial s} f\left(\frac{b^{\rho_{1}}}{m}, \frac{c^{\rho_{2}}}{m}\right)\right|^{q}\right)^{\frac{1}{q}} \\
& +\left(\left|\frac{\partial^{2}}{\partial t \partial s} f\left(a^{\rho_{1}}, c^{\rho_{2}}\right)\right|^{q}+\alpha \rho_{2} m\left|\frac{\partial^{2}}{\partial t \partial s} f\left(a^{\rho_{1}}, \frac{d^{\rho_{2}}}{m}\right)\right|^{q}\right. \\
& \left.\left.+\alpha \rho_{1} m\left|\frac{\partial^{2}}{\partial t \partial s} f\left(\frac{b^{\rho_{1}}}{m}, c^{\rho_{2}}\right)\right|^{q}+\alpha^{2} m^{2} \rho_{1} \rho_{2}\left|\frac{\partial^{2}}{\partial t \partial s} f\left(\frac{b^{\rho_{1}}}{m}, \frac{d^{\rho_{2}}}{m}\right)\right|^{q}\right)^{\frac{1}{q}}\right\},
\end{aligned}
$$

where $\frac{1}{r}+\frac{1}{q}=1$. 
Proof. By using Lemma 2.4 and the properties of the absolute value, we obtain

$$
\begin{aligned}
& \frac{f\left(a^{\rho_{1}, c^{\rho}}\right)+f\left(a^{\rho_{1}, d^{\rho} 2}\right)+f\left(b^{\rho_{1}}, c^{\rho_{2}}\right)+f\left(b^{\left.\rho_{1}, d^{\rho_{2}}\right)}\right.}{4} \\
& -\frac{\rho_{2}^{\beta_{2}} \Gamma\left(\beta_{2}+1\right)}{4\left(d^{\rho_{2}}-c^{\rho_{2}}\right)^{\beta_{2}}}\left[\rho_{2} I_{c+}^{\beta_{2}} f\left(a^{\rho_{1}}, d^{\rho_{2}}\right)+{ }^{\rho_{2}} I_{c+}^{\beta_{2}} f\left(b^{\rho_{1}}, d^{\rho_{2}}\right)\right. \\
& \left.+{ }^{\rho_{2}} I_{d-}^{\beta_{2}} f\left(a^{\rho_{1}}, c^{\rho_{2}}\right)+{ }^{\rho_{2}} I_{d-}^{\beta_{2}} f\left(b^{\rho_{1}}, c^{\rho_{2}}\right)\right] \\
& -\frac{\rho_{1}^{\beta_{1}} \Gamma\left(\beta_{1}+1\right)}{4\left(b^{\rho_{1}}-a^{\rho_{1}}\right)^{\beta_{1}}}\left[{ }^{\rho_{1}} I_{a+}^{\beta_{1}} f\left(b^{\rho_{1}}, c^{\rho_{2}}\right)+{ }^{\rho_{1}} I_{a+}^{\beta_{1}} f\left(b^{\rho_{1}}, d^{\rho_{2}}\right)\right. \\
& \left.+{ }^{\rho_{1}} I_{b-}^{\beta_{1}} f\left(a^{\rho_{1}}, c^{\rho_{2}}\right)+{ }^{\rho_{1}} I_{b-}^{\beta_{1}} f\left(a^{\rho_{1}}, d^{\rho_{2}}\right)\right] \\
& +\frac{\rho_{1}^{\beta_{1}} \rho_{2}^{\beta_{2}} \Gamma\left(\beta_{1}+1\right) \Gamma\left(\beta_{2}+1\right)}{4\left(b^{\rho_{1}}-a^{\rho_{1}}\right)^{\beta_{1}}\left(d^{\left.\rho_{2}-c^{\rho_{2}}\right)^{\beta_{2}}}\right.}\left[{ }^{\rho_{1}, \rho_{2}} I_{a+, c+}^{\beta_{1}, \beta_{2}} f\left(b^{\rho_{1}}, d^{\rho_{2}}\right)+{ }^{\rho_{1}, \rho_{2}} I_{a+, d-}^{\beta_{1}, \beta_{2}} f\left(b^{\rho_{1}}, c^{\rho_{2}}\right)\right. \\
& \left.+{ }^{\rho_{1}, \rho_{2}} I_{b-, c+}^{\beta_{1}, \beta_{2}} f\left(c^{\rho_{1}}, d^{\rho_{2}}\right)+{ }^{\rho_{1}, \rho_{2}} I_{b-, d-}^{\beta_{1}, \beta_{2}} f\left(a^{\rho_{1}}, c^{\rho_{2}}\right)\right] \mid \\
& \leq \frac{\rho_{1} \rho_{2}\left(b^{\rho_{1}}-a^{\rho_{1}}\right)\left(d^{\left.\rho_{2}-c^{\rho_{2}}\right)}\right.}{4}\left(\left|I_{1}\right|+\left|I_{2}\right|+\left|I_{3}\right|+I_{4} \mid\right) .
\end{aligned}
$$

By using the Hölder's inequality and the $(\alpha, m)$-convexity of $\left|\frac{\partial^{2} f}{\partial t \partial s}\right|^{q}$ on the coordinates, we have

$$
\begin{aligned}
\left|I_{1}\right| \leq & \left(\int_{0}^{1} \int_{0}^{1} s^{\left(\beta_{2}+1\right) \rho_{2} r-r} t^{\left(\beta_{1}+1\right) \rho_{1} r-r} d s d t\right)^{\frac{1}{r}} \\
& \times\left(\int_{0}^{1} \int_{0}^{1}\left|\frac{\partial^{2}}{\partial t \partial s} f\left(t^{\rho_{1}} b^{\rho_{1}}+\left(1-t^{\rho_{1}}\right) a^{\rho_{1}}, s^{\rho_{2}} d^{\rho_{2}}+\left(1-s^{\rho_{2}}\right) c^{\rho_{2}}\right)\right|^{q} d t d s\right)^{\frac{1}{q}} \\
\leq & \left(\frac{1}{\left(\left(\beta_{1}+1\right) \rho_{1} r-r+1\right)\left(\left(\beta_{2}+1\right) \rho_{2} r-r+1\right)}\right)^{\frac{1}{r}} \\
& \times\left(\left|\frac{\partial^{2}}{\partial t \partial s} f\left(b^{\rho_{1}}, d^{\rho_{2}}\right)\right|^{q} \int_{0}^{1} \int_{0}^{1} s^{\alpha \rho_{2}} t^{\alpha \rho_{1}} d t d s\right.
\end{aligned}
$$




$$
\begin{aligned}
& +m\left|\frac{\partial^{2}}{\partial t \partial s} f\left(b^{\rho_{1}}, \frac{c^{\rho_{2}}}{m}\right)\right|^{q} \int_{0}^{1} \int_{0}^{1}\left(1-s^{\alpha \rho_{2}}\right) t^{\alpha \rho_{1}} d t d s \\
& +\left.m\left|\frac{\partial^{2}}{\partial t \partial s} f\left(\frac{a^{\rho_{1}}}{m}, d^{\rho_{2}}\right)\right|\right|_{0} ^{q} \int_{0}^{1} s^{\alpha \rho_{2}}\left(1-t^{\alpha \rho_{1}}\right) d t d s \\
& \left.+m^{2}\left|\frac{\partial^{2}}{\partial t \partial s} f\left(\frac{a^{\rho_{1}}}{m}, \frac{c^{\rho_{2}}}{m}\right)\right|^{q} \int_{0}^{1} \int_{0}^{1}\left(1-s^{\alpha \rho_{2}}\right)\left(1-t^{\alpha \rho_{1}}\right) d t d s\right)^{\frac{1}{q}} \\
& =\left(\frac{1}{\left(\left(\beta_{1}+1\right) \rho_{1} r-r+1\right)\left(\left(\beta_{2}+1\right) \rho_{2} r-r+1\right)}\right)^{\frac{1}{r}}\left(\frac{1}{\left(\alpha \rho_{1}+1\right)\left(\alpha \rho_{2}+1\right)}\left|\frac{\partial^{2}}{\partial t \partial s} f\left(b^{\rho_{1}}, d^{\rho_{2}}\right)\right|^{q}\right. \\
& +\left.\frac{1}{\left(\alpha \rho_{1}+1\right)}\left(\frac{\alpha \rho_{2}}{\left(\alpha \rho_{2}+1\right)}\right) m\left|\frac{\partial^{2}}{\partial t \partial s} f\left(b^{\rho_{1}}, \frac{c^{\rho_{2}}}{m}\right)\right|^{q}\right|^{q} \\
& +\frac{1}{\left(\alpha \rho_{2}+1\right)}\left(\frac{\alpha \rho_{1}}{\left(\alpha \rho_{1}+1\right)}\right) m\left|\frac{\partial^{2}}{\partial t \partial s} f\left(\frac{a^{\rho_{1}}}{m}, d^{\rho_{2}}\right)\right|^{q} \\
& \left.+\left(\frac{\alpha \rho_{2}}{\left(\alpha \rho_{2}+1\right)}\right)\left(\frac{\alpha \rho_{1}}{\left(\alpha \rho_{1}+1\right)}\right) m^{2}\left|\frac{\partial^{2}}{\partial t \partial s} f\left(\frac{a^{\rho_{1}}}{m}, \frac{c^{\rho_{2}}}{m}\right)\right|^{q}\right)^{\frac{1}{q}} .
\end{aligned}
$$

Thus,

$$
\begin{aligned}
\left|I_{1}\right| \leq & \left.\left(\frac{1}{\left(\left(\beta_{1}+1\right) \rho_{1} r-r+1\right)\left(\left(\beta_{2}+1\right) \rho_{2} r-r+1\right)}\right)\right)^{\frac{1}{r}}\left(\frac{1}{\left(\alpha \rho_{1}+1\right)\left(\alpha \rho_{2}+1\right)}\right)^{\frac{1}{q}} \\
& \times\left(\left|\frac{\partial^{2}}{\partial t \partial s} f\left(b^{\rho_{1}}, d^{\rho_{2}}\right)\right|^{q}+\alpha \rho_{2} m\left|\frac{\partial^{2}}{\partial t \partial s} f\left(b^{\rho_{1}}, \frac{c^{\rho_{2}}}{m}\right)\right|^{q}\right. \\
& \left.+\alpha \rho_{1} m\left|\frac{\partial^{2}}{\partial t \partial s} f\left(\frac{a^{\rho_{1}}}{m}, d^{\rho_{2}}\right)\right|^{q}+\alpha^{2} m^{2} \rho_{1} \rho_{2}\left|\frac{\partial^{2}}{\partial t \partial s} f\left(\frac{a^{\rho_{1}}}{m}, \frac{c^{\rho_{2}}}{m}\right)\right|^{q}\right)^{\frac{1}{q}} .
\end{aligned}
$$

Using similar arguments, we have

$$
\begin{aligned}
\left|I_{2}\right| \leq & \left(\frac{1}{\left(\left(\beta_{1}+1\right) \rho_{1} r-r+1\right)\left(\left(\beta_{2}+1\right) \rho_{2} r-r+1\right)}\right)^{\frac{1}{r}}\left(\frac{1}{\left(\alpha \rho_{1}+1\right)\left(\alpha \rho_{2}+1\right)}\right)^{\frac{1}{q}} \\
& \times\left(\left|\frac{\partial^{2}}{\partial t \partial s} f\left(b^{\rho_{1}}, c^{\rho_{2}}\right)\right|^{q}+\alpha \rho_{2} m\left|\frac{\partial^{2}}{\partial t \partial s} f\left(b^{\rho_{1}}, \frac{d^{\rho_{2}}}{m}\right)\right|^{q}\right. \\
& \left.+\alpha \rho_{1} m\left|\frac{\partial^{2}}{\partial t \partial s} f\left(\frac{a^{\rho_{1}}}{m}, c^{\rho_{2}}\right)\right|^{q}+\alpha^{2} m^{2} \rho_{1} \rho_{2}\left|\frac{\partial^{2}}{\partial t \partial s} f\left(\frac{a^{\rho_{1}}}{m}, \frac{d^{\rho_{2}}}{m}\right)\right|^{q}\right)^{\frac{1}{q}}
\end{aligned}
$$




$$
\begin{aligned}
\left|I_{3}\right| \leq & \left(\frac{1}{\left(\left(\beta_{1}+1\right) \rho_{1} r-r+1\right)\left(\left(\beta_{2}+1\right) \rho_{2} r-r+1\right)}\right)^{\frac{1}{r}}\left(\frac{1}{\left(\alpha \rho_{1}+1\right)\left(\alpha \rho_{2}+1\right)}\right)^{\frac{1}{q}} \\
& \times\left(\left|\frac{\partial^{2}}{\partial t \partial s} f\left(a^{\rho_{1}}, d^{\rho_{2}}\right)\right|^{q}+\alpha \rho_{2} m\left|\frac{\partial^{2}}{\partial t \partial s} f\left(a^{\rho_{1}}, \frac{c^{\rho_{2}}}{m}\right)\right|^{q}\right. \\
& \left.+\alpha \rho_{1} m\left|\frac{\partial^{2}}{\partial t \partial s} f\left(\frac{b^{\rho_{1}}}{m}, d^{\rho_{2}}\right)\right|^{q}+\alpha^{2} m^{2} \rho_{1} \rho_{2}\left|\frac{\partial^{2}}{\partial t \partial s} f\left(\frac{b^{\rho_{1}}}{m}, \frac{c^{\rho_{2}}}{m}\right)\right|^{q}\right)^{\frac{1}{q}}
\end{aligned}
$$

and

$$
\begin{aligned}
\left|I_{4}\right| \leq & \left(\frac{1}{\left(\left(\beta_{1}+1\right) \rho_{1} r-r+1\right)\left(\left(\beta_{2}+1\right) \rho_{2} r-r+1\right)}\right)^{\frac{1}{r}}\left(\frac{1}{\left(\alpha \rho_{1}+1\right)\left(\alpha \rho_{2}+1\right)}\right)^{\frac{1}{q}} \\
& \times\left(\left|\frac{\partial^{2}}{\partial t \partial s} f\left(a^{\rho_{1}}, c^{\rho_{2}}\right)\right|^{q}+\alpha \rho_{2} m\left|\frac{\partial^{2}}{\partial t \partial s} f\left(a^{\rho_{1}}, \frac{d^{\rho_{2}}}{m}\right)\right|^{q}\right. \\
& \left.+\alpha \rho_{1} m\left|\frac{\partial^{2}}{\partial t \partial s} f\left(\frac{b^{\rho_{1}}}{m}, c^{\rho_{2}}\right)\right|^{q}+\alpha^{2} m^{2} \rho_{1} \rho_{2}\left|\frac{\partial^{2}}{\partial t \partial s} f\left(\frac{b^{\rho_{1}}}{m}, \frac{d^{\rho_{2}}}{m}\right)\right|^{q}\right)^{\frac{1}{q}} .
\end{aligned}
$$

The desired inequality follows from (2.17) and using (2.18)-(2.21).

\section{Conclusion}

We established a new integral inequality of Hermite-Hadamard type for functions of two variables that are $m$-convex on the coordinates via generalized Katugampola type fractional integrals of functions of two variables. We also established three new integral inequalities of Hermite-Hadamard type for functions whose second order mixed partial derivatives in absolute value at certain powers are $(\alpha, m)$-convex on the coordinates. If we choose $(\alpha, m)=(1,1)$, then our results hold for functions whose second order mixed partial derivatives are convex on the coordinates. We believe that, results in this paper we inspire further research on the inequalities involving functions of two independent variables via generalized fractional integrals. 


\section{References}

[1] M. K. Bakula, M. E. Özdemir, and J. Pečarić, "Hadamard-type inequalities for m-convex and $(\alpha, \mathrm{m})$-convex functions", Journal of inequalities in pure and applied mathematics, vol. 9, no. 4, Art. ID. 96, 2008.

[2] H. Chen and U. N. Katugampola, "Hermite-Hadamard and HermiteHadamard-Fejér type inequalities for generalized fractional integrals", Journal of mathematical analysis and applications, vol. 446, no. 2, pp. 1274-1291, 2017. https:/ / doi.org/ g6rr

[3] S. S. Dragomir, "On the Hadamard's inequlality for convex functions on the co-ordinates in a rectangle from the plane", Taiwanese journal of mathematics, vol. 5, no. 4, pp. 775-788, 2001. https:/ / doi.org/ g6rs

[4] D.-Y. Hwang, K.-L. Tseng, and G.-S. Yang, "Some Hadamard's inequalities for co-ordinated convex functions in a rectangle from the plane," Taiwanese journal of mathematics, vol. 11, no. 1, pp. 63-73, 2007. https:/ / doi.org/ 10.11650/ twjm/ 1500404635

[5] U. N. Katugampola, "New approach to a generalized fractional integral," Applied mathematics and computation, vol. 218, no. 3, pp. 860-865, 2011. https:/ / doi.org/ 10.1016/j.amc.2011.03.062

[6] U. N. Katugampola, "A new approach to generalized fractional derivatives", Bulletin of mathematical analysis and applications, vol. 6, no. 4, pp. 1-15, 2014.

[7] S. Kermausuor, "Ostrowski type inequalities for functions whose derivatives are strongly $(\mathrm{a}, \mathrm{m})$-convex via K-riemann-liouville fractional integrals", Studia Universitatis Babes-Bolyai Matematica, vol. 64, no. 1, pp. 25-34, 2019. https:/ / doi.org/ 10.24193/ subbmath.2019.1.03

[8] S. Kermausuor, "Generalized Ostrowski-type inequalities involving second derivatives via the Katugampola fractional integrals", Journal of nonlinear sciences and applications, vol. 12, no. 08, pp. 509-522, 2019. https:/ / doi.org/ 10.22436/jnsa.012.08.02

[9] S. Kermausuor, "Simpson's type inequalities via the Katugampola fractional integrals for s-convex functions", Kragujevac journal of mathematics, vol. 45, no. 5, pp. 709-720, 2021. 
[10] S. Kermausuor and E. R. Nwaeze, "Some new inequalities involving the Katugampola fractional integrals for strongly n-convex functions", Tbilisi mathematical journal, vol. 12, no. 1, pp. 117-130, 2019. https:/ / doi.org/ 10.32513/ tbilisi/ 1553565631

[11] S. Kermausuor, E. R. Nwaeze, and A. M. Tameru, "New integral inequalities via the katugampola fractional integrals for functions whose second derivatives are strongly $\eta$-convex", Mathematics, vol. 7, no. 2, 183, 2019. https:/ / doi.org/ 10.3390/ math7020183

[12] M. A. Latif, "New Ostrowski type inequalities for co-ordinated ( $\alpha, \mathrm{m})$ convex functions", Transylvanian journal of mathematics and mechanics, vol. 6, no. 2, pp. 139-150, 2014.

[13] M. W. Alomari, M. A. Latif, and S. Hussain, "On ostrowski-type inequalities for functions whose derivatives are M-convex and $(\alpha$, m)-convex functions with applications", Tamkang journal of mathematics, vol. 43, no. 4, 2012. https:// doi.org/ g6r9

[14] M . E. Özdemir, M. A vci, and H. K avurmaci, "H ermite-H adamardtype inequalities via $(\alpha, m)$ - convexity", Computers $\&$ mathematics with applications, vol. 61, no. 9, pp. 2614-2620, 2011. https:/ / doi.org/ dp6ffs

[15] M. E. Özdemir, H. Kavurmaci, and E. Set, "Ostrowski type inequalities for $(\alpha, \mathrm{m})$-convex functions", Kyungpook mathematical journal, vol. 50, no. 3, pp. 371-378, 2010.

[16] M. E. Özdemir, E. Set, and M. Z Sarikaya, "Some new Hadamard's type inequalities for co-ordinated m-convex and $(\alpha, \mathrm{m})$-convex functions", Hacettepe journal of mathematics and statistics, vol. 40, no. 2, pp. 219229, 2011, https:/ / bit.ly/ 3nEUmW0

[17] W. Sun and Q. Liu, "New Hermite-Hadamard type inequalities for ( $\alpha$, $\mathrm{m}$ )-convex functions and applications to special means", Journal of mathematical inequalities, vol. 11, no. 2, pp. 383-397, 2017. https:/ / doi.org/ 10.7153/jmi-11-33

\section{Seth Kermausuor}

Department of Mathematics and Computer Science, Alabama State University,

Montgomery, AL 36101,

U. S. A.

e-mail: skermausour@alasu.edu 